Article

\title{
Sesquiterpenoids and Xanthones from the Kiwifruit-Associated Fungus Bipolaris sp. and Their Anti-Pathogenic Microorganism Activity
}

\author{
Jun-Jie Yu $(D)$ Ying-Xue Jin, Shan-Shan Huang and Juan He *D \\ National Demonstration Center for Experimental Ethnopharmacology Education, School of Pharmaceutical \\ Sciences, South-Central University for Nationalities, Wuhan 430074, China; junjieyu98@outlook.com (J.-J.Y.); \\ Yancy6020@163.com (Y.-X.J.); HuangSS1998@126.com (S.-S.H.) \\ * Correspondence: 2015048@mail.scuec.edu.cn
}

Citation: Yu, J.-J.; Jin, Y.-X.; Huang, S.-S.; He, J. Sesquiterpenoids and Xanthones from the KiwifruitAssociated Fungus Bipolaris sp. and Their Anti-Pathogenic

Microorganism Activity. J. Fungi 2022, 8,9. https://doi.org/10.3390/ jof8010009

Academic Editor: Frank Surup

Received: 13 December 2021

Accepted: 21 December 2021

Published: 23 December 2021

Publisher's Note: MDPI stays neutral with regard to jurisdictional claims in published maps and institutional affiliations.

Copyright: (C) 2021 by the authors. Licensee MDPI, Basel, Switzerland. This article is an open access article distributed under the terms and conditions of the Creative Commons Attribution (CC BY) license (https:// creativecommons.org/licenses/by/ $4.0 /)$.
Abstract: Nine previously undescribed sesquiterpenoids, bipolarisorokins A-I (1-9); two new xanthones, bipolarithones $\mathrm{A}$ and $\mathrm{B}$ (10 and 11); two novel sativene-xanthone adducts, bipolarithones $\mathrm{C}$ and D (12 and 13); as well as five known compounds (14-18) were characterized from the kiwifruitassociated fungus Bipolaris sp. Their structures were elucidated by extensive spectroscopic methods, electronic circular dichroism (ECD), ${ }^{13} \mathrm{C}$ NMR calculations, DP4+ probability analyses, and single crystal X-ray diffractions. Many compounds exhibited anti-pathogenic microorganism activity against the bacterium Pseudomonas syringae pv. actinidiae and four pathogenic microorganisms.

Keywords: Bipolaris sp.; kiwi-associated fungus; sesquiterpenoid; xanthone; anti-pathogenic microorganism activity

\section{Introduction}

Kiwifruit (Actinidia chinensis Planch., Actinidiaceae) is an emerging, healthy, and economical fruit which has become increasingly popular worldwide owing to its flavor and nutritional properties [1]. It is an excellent source of vitamin $C$ and provides balanced nutritional components of minerals, dietary fiber, folate, and health-promoting metabolites [2,3]. China is the leading kiwifruit producer in the world, followed by Italy and New Zealand. The cultivation area and annual output reached $243,000 \mathrm{hm}^{2}$ and 2,500,000 tons at the end of 2020 [4]. Nevertheless, as the cultivation of kiwifruit expands rapidly, many serious diseases such as bacterial canker, soft rot, bacterial blossom blight, brown spot, and root rot are a serious and ongoing threat to kiwifruit production [5-12]. Particularly, the destructive bacterial canker disease, which is associated with an infection by $P$. syringae pv. actinidiae (Psa), has led to reduced kiwifruit production and huge economic losses worldwide $[13,14]$. Although the application of copper-based chemicals and streptomycin have played a positive role in the prevention and treatment of bacterial canker, these chemical residues are extremely threatening to human health and the ecological environment $[15,16]$. Additionally, chemical fungicides easily induce pathogen resistance $[17,18]$. Thus, it is urgent to develop safer and more effective biological pesticides.

Endophytic microorganisms reside within different tissues of the host plant without causing any disease symptoms and produce various metabolites with different activities $[19,20]$. Therefore, the endophytic fungi have been proved to be valuable sources of important natural products $[21,22]$. Some natural products from endophytic fungi play important roles in plant defense systems. Therefore, we carried out the excavation of anti-Psa active substances from metabolites of kiwifruit endophytes and harvested a number of bioactive molecules. For instance, 3-decalinoyltetramic acids and cytochalasins from the kiwifruit endophytic fungus Zopfiella sp showed anti-Psa activity $[23,24]$, while imidazole alkaloids ether were characterized as anti-Psa agents from Fusarium tricinctum [25]. These 
discoveries prompted us to search for more novel and bioactive metabolites from kiwifruitassociated fungi. In the current study, a total of eighteen compounds have been isolated from the large-scale fermentation of the kiwifruit-associated fungus Bipolaris sp. (Figure 1), which included nine new sativene or longifolene sesquiterpenoids, bipolarisorokins A-I (1-9); two new xanthones, bipolarithones $A$ and $B$ (10 and 11); two novel sativene-xanthone adducts, bipolarithones $C$ and D (12 and 13); as well as five known ones (14-18). Their structures were established by means of spectroscopic methods, namely, ECD and ${ }^{13} \mathrm{C}$ NMR calculations, DP4+ probability analyses, and single crystal X-ray diffractions. All compounds were evaluated for their inhibitory activities against Psa. Additionally, their inhibitory activity against four phytopathogens (Phytophthora infestans, Alternaria solani, Rhizoctonia solani, and Fusarium oxysporum) were assessed. Here, the details of isolation, structural elucidation, and bioactivity evaluations for 1-18 are reported.

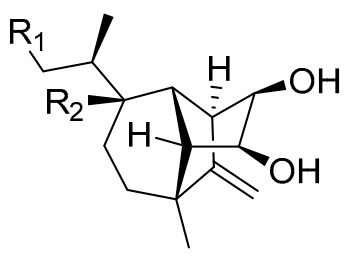

$1 \mathrm{R}_{1}=\mathrm{OH}, \mathrm{R}_{2}=\mathrm{H}$

$2 \mathrm{R}_{1}=\mathrm{H}, \mathrm{R}_{2}=\mathrm{OH}$

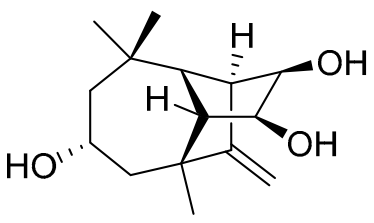

9<smiles></smiles>

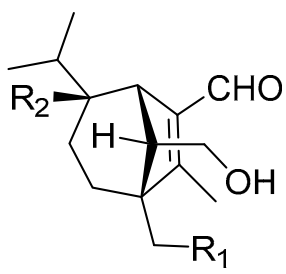

$3 \mathrm{R}_{1}=\mathrm{OH}, \mathrm{R}_{2}=\mathrm{H}$

$4 \mathrm{R}_{1}=\mathrm{H}, \mathrm{R}_{2}=\mathrm{OH}$

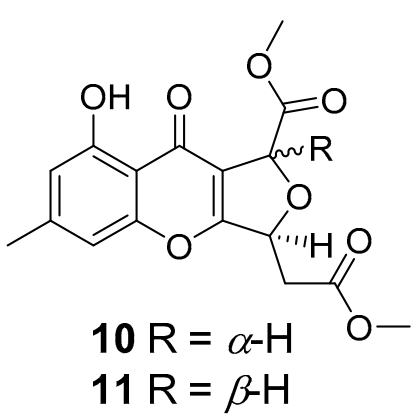<smiles>C=C(C)[C@H]1CCC2(C)CCC1C=C2CO</smiles>

5

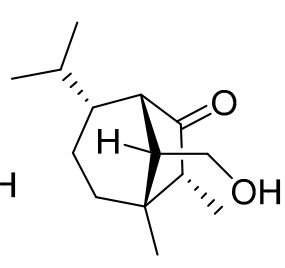

6

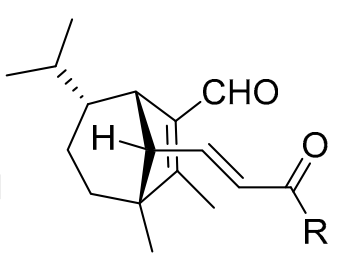

$7 \mathrm{R}=\mathrm{CH}_{3}$

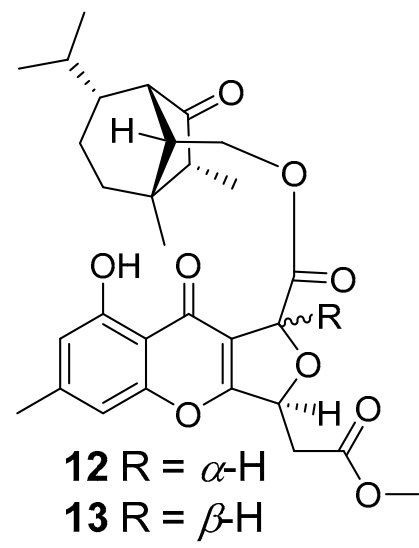

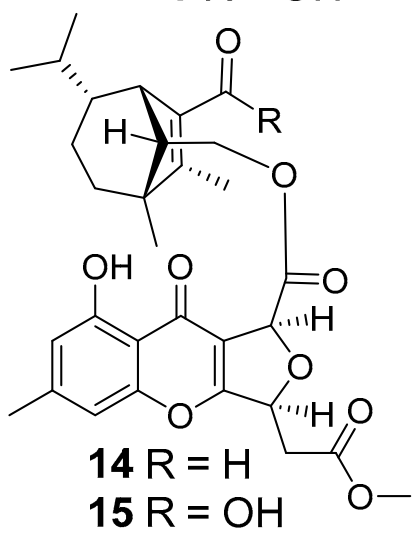

Figure 1. Structures of compounds 1-18.

\section{Materials and Methods}

\subsection{General Experimental Procedures}

Melting points were obtained on an X-4 micro melting point apparatus. Optical rotations were measured with an Autopol IV polarimeter (Rudolph, Hackettstown, NJ, USA). UV spectra were obtained using a double beam spectrophotometer UH5300 (Hitachi High-Technologies, Tokyo, Japan). IR spectra were obtained by a Shimadzu IRTracer-100 spectrometer using $\mathrm{KBr}$ pellets. 1D and 2D NMR spectra were run on a Bruker Avance III $600 \mathrm{MHz}$ spectrometer with TMS as an internal standard. Chemical shifts $(\delta)$ were expressed in ppm with references to the solvent signals. High resolution electrospray ionization mass spectra (HR-ESIMS) were recorded on a LC-MS system consisting of a Q Exactive ${ }^{\mathrm{TM}} \mathrm{Or}-$ bitrap mass spectrometer with an HRESI ion source (ThermoFisher Scientific, Bremen, Germany) used in ultra-high-resolution mode (140,000 at m/z 200) and a UPLC system 
(Dionex UltiMate 3000 RSLC, ThermoFisher Scientific, Bremen, Germany). Column chromatography (CC) was performed on silica gel (200-300 mesh, Qingdao Marine Chemical Ltd., Qingdao, China), RP-18 gel (20-45 $\mu$ m, Fuji Silysia Chemical Ltd., Kasugai, Japan), and Sephadex LH-20 (Pharmacia Fine Chemical Co. Ltd., Uppsala, Sweden). Medium-pressure liquid chromatography (MPLC) was performed on a Büchi Sepacore System equipped with a pump manager C-615, pump modules C-605, and a fraction collector C-660 (Büchi Labortechnik AG, Flawil, Switzerland). Preparative high-performance liquid chromatography (prep-HPLC) was performed on an Agilent 1260 liquid chromatography system equipped with Zorbax SB-C18 columns $(5 \mu \mathrm{m}, 9.4 \mathrm{~mm} \times 150 \mathrm{~mm}$, or $21.2 \mathrm{~mm} \times 150 \mathrm{~mm})$ and a DAD detector. Chiral resolution was achieved by HPLC equipped with a Daicel AD-H column. Fractions were monitored by TLC (GF 254, Qingdao Haiyang Chemical Co. Ltd. Qingdao, China), and spots were visualized by heating silica gel plates sprayed with $10 \% \mathrm{H}_{2} \mathrm{SO}_{4}$ in $\mathrm{EtOH}$.

\subsection{Fermentation, Extraction, and Isolation}

The fungus Bipolaris sp. was isolated from fresh and healthy stems of kiwifruit plants (Actinidia chinensis Planch., Actinidiaceae), which were collected from the Cangxi county of the Sichuan Province (GPS: N 31 ${ }^{\circ} 12^{\prime}$, E $105^{\circ} 76^{\prime}$ ) in July 2018. Each fungus was obtained simultaneously from at least three different healthy tissues. The fungus was identified as one species of the genus Bipolaris by observing the morphological characteristics and analysis of the internal transcribed spacer (ITS) regions. A living culture (internal number HFG-20180727-HJ32) has been deposited at the School of Pharmaceutical Sciences, SouthCentral University for Nationalities, China.

This fungal strain was cultured on a potato dextrose agar (PDA) medium at $24{ }^{\circ} \mathrm{C}$ for 10 days. The agar plugs were inoculated in $500 \mathrm{~mL}$ Erlenmeyer flasks, each containing $100 \mathrm{~mL}$ potato dextrose media. Flask cultures were incubated at $28^{\circ} \mathrm{C}$ on a rotary shaker at $160 \mathrm{rpm}$ for two days as the seed culture. Four hundred $500 \mathrm{~mL}$ Erlenmeyer flasks, each containing $150 \mathrm{~mL}$ potato dextrose broth (PDB), were individually inoculated with $25 \mathrm{~mL}$ of seed culture and were incubated at $25^{\circ} \mathrm{C}$ on a rotary shaker at $160 \mathrm{rpm}$ for 25 days.

The cultures of Bipolaris sp. (60 L) were extracted four times by EtOAc to afford a crude extract $(32.0 \mathrm{~g})$ which was subjected to $\mathrm{CC}$ over silica gel eluted with a gradient of $\mathrm{CHCl}_{3}-\mathrm{MeOH}$ (a gradient from 1:0 to 0:1) to give six fractions, A-F. Fraction B (13.0 g) was fractionated by MPLC CC over RP-18 eluted with $\mathrm{MeOH}-\mathrm{H}_{2} \mathrm{O}$ (from 10:90 to 100:0, $v / v)$ to give twelve sub-fractions $\left(\mathrm{B}_{1}-\mathrm{B}_{12}\right)$. Fraction $\mathrm{B}_{3}(1.2 \mathrm{~g})$ was applied to Sephadex LH-20 eluting with $\mathrm{CHCl}_{3}-\mathrm{MeOH}(1: 1, v / v)$ and was further purified by preparative HPLC with $\mathrm{MeCN}-\mathrm{H}_{2} \mathrm{O}(19: 81, v / v, 4.0 \mathrm{~mL} / \mathrm{min})$ to obtain compounds 9 (18.6 $\mathrm{mg}$, retention time $\left.\left(t_{R}\right)=40 \mathrm{~min}\right), 18\left(22.6 \mathrm{mg}, t_{R}=15.8 \mathrm{~min}\right), 2\left(3.3 \mathrm{mg}, \mathrm{t}_{\mathrm{R}}=32 \mathrm{~min}\right)$, and $\mathbf{1}\left(5.4 \mathrm{mg}, \mathrm{t}_{\mathrm{R}}=36\right.$ $\mathrm{min})$. Fraction $\mathrm{B}_{5}(2.1 \mathrm{~g})$ was separated by $\mathrm{CC}$ over silica gel with a gradient elution of the $\mathrm{CHCl}_{3}-\mathrm{MeOH}$ system $(50: 1 \rightarrow 0: 1)$ and was prepared by HPLC with $\mathrm{MeCN}-\mathrm{H}_{2} \mathrm{O}(12: 88$, $v / v, 4.0 \mathrm{~mL} / \mathrm{min})$ to obtain $3\left(4.9 \mathrm{mg}, \mathrm{t}_{\mathrm{R}}=36 \mathrm{~min}\right), 4\left(14.4 \mathrm{mg}, \mathrm{t}_{\mathrm{R}}=46 \mathrm{~min}\right), \mathbf{1 7}(28.3 \mathrm{mg}$, $\left.\mathrm{t}_{\mathrm{R}}=43 \mathrm{~min}\right)$, and $5\left(2.1 \mathrm{mg}, \mathrm{t}_{\mathrm{R}}=40 \mathrm{~min}\right)$. Fraction $\mathrm{B}_{6}(1.8 \mathrm{~g})$ was purified over Sephadex LH-20 eluted with $\mathrm{MeOH}$ to give four subfractions $\left(\mathrm{B}_{6.1}-\mathrm{B}_{6.4}\right)$. Fraction $\mathrm{B}_{6.2}(210 \mathrm{mg})$ was purified using semipreparative $\mathrm{HPLC}$ with $\mathrm{MeOH}-\mathrm{H}_{2} \mathrm{O}(28: 72, v / v, 3.0 \mathrm{~mL} / \mathrm{min})$ to afford $8\left(8.8 \mathrm{mg}, \mathrm{t}_{\mathrm{R}}=17.8 \mathrm{~min}\right)$ and $7\left(9.6 \mathrm{mg}, \mathrm{t}_{\mathrm{R}}=21.1 \mathrm{~min}\right)$. Fraction $\mathrm{B}_{6.3}(170 \mathrm{mg})$ was purified by preparative $\mathrm{HPLC}$ with $\mathrm{MeCN}-\mathrm{H}_{2} \mathrm{O}(23: 77, v / v, 4 \mathrm{~mL} / \mathrm{min})$ to yield $6(4.3 \mathrm{mg}, 26 \mathrm{~min})$. Fraction $\mathrm{C}(4.3 \mathrm{~g})$ was separated by CC over silica gel with a gradient elution of PE-acetone $(50: 1 \rightarrow 0: 1)$ to afford subfractions $\mathrm{C}_{1}-\mathrm{C}_{8}$. Fraction $\mathrm{C}_{2}(340 \mathrm{mg})$ was purified by preparative $\mathrm{HPLC}$ with $\mathrm{MeCN}-\mathrm{H}_{2} \mathrm{O}(55: 45, v / v, 4 \mathrm{~mL} / \mathrm{min})$ to give $12\left(10.3 \mathrm{mg}, \mathrm{t}_{\mathrm{R}}=38 \mathrm{~min}\right), \mathbf{1 3}(3.7 \mathrm{mg}$, $\left.\mathrm{t}_{\mathrm{R}}=39 \mathrm{~min}\right), \mathbf{1 4}\left(3.1 \mathrm{mg}, \mathrm{t}_{\mathrm{R}}=36 \mathrm{~min}\right)$ and $15\left(3.4 \mathrm{mg}, \mathrm{t}_{\mathrm{R}}=34 \mathrm{~min}\right)$. Fraction $\mathrm{C}_{5}(230 \mathrm{mg})$ was isolated by CC over Sephadex LH-20 $(\mathrm{MeOH})$ and was prepared by HPLC (32:68, $v / v, 4 \mathrm{~mL} / \mathrm{min})$ to give $\mathbf{1 0}\left(3.7 \mathrm{mg}, \mathrm{t}_{\mathrm{R}}=28 \mathrm{~min}\right), \mathbf{1 1}\left(4.2 \mathrm{mg}, \mathrm{t}_{\mathrm{R}}=29 \mathrm{~min}\right)$, and $\mathbf{1 6}(5.1 \mathrm{mg}$, $\left.\mathrm{t}_{\mathrm{R}}=24 \mathrm{~min}\right)$.

Bipolarisorokin A (1): colorless crystals; mp $145-148{ }^{\circ} \mathrm{C} ;[\alpha]^{20} \mathrm{D}+67.8(c 0.01, \mathrm{MeOH})$; UV (MeOH) $\lambda_{\max }(\log \varepsilon) 205$ (3.30); IR (KBr) $v_{\max }$ 3360, 2947, 2833, 1651, 1454, 1114, 
$1031 \mathrm{~cm}^{-1} ;{ }^{1} \mathrm{H}$ NMR (600 MHz, $\left.\mathrm{CDCl}_{3}\right)$ and ${ }^{13} \mathrm{C} \mathrm{NMR}\left(150 \mathrm{MHz}, \mathrm{CDCl}_{3}\right)$ data, see Table 1; positive ion HRESIMS $m / z 251.16624[\mathrm{M}-\mathrm{H}]^{+}$, (calculated for $\mathrm{C}_{15} \mathrm{H}_{23} \mathrm{O}_{3}{ }^{-}$251.16527).

Table 1. ${ }^{1} \mathrm{H}(600 \mathrm{MHz})$ and ${ }^{13} \mathrm{C}(150 \mathrm{MHz}) \mathrm{NMR}$ Spectroscopic Data for $\mathbf{1}-\mathbf{3}$.

\begin{tabular}{|c|c|c|c|c|c|c|}
\hline \multirow{2}{*}{ No. } & \multicolumn{2}{|c|}{$1^{a}$} & \multicolumn{2}{|c|}{$2^{a}$} & \multicolumn{2}{|r|}{$3^{b}$} \\
\hline & $\delta_{\mathrm{C}}$, Type & $\delta_{\mathrm{H}}(J$ in $\mathrm{Hz})$ & $\delta_{\mathrm{C}}$, Type & $\delta_{\mathrm{H}}(J$ in $\mathrm{Hz})$ & $\delta_{\mathrm{C}}$, Type & $\delta_{\mathrm{H}}(J$ in $\mathrm{Hz})$ \\
\hline 1 & $54.3, \mathrm{CH}$ & $2.71, \mathrm{~s}$ & $55.4, \mathrm{CH}$ & 2.59, br s & $140,0, \mathrm{C}$ & \\
\hline 2 & $156.8, \mathrm{C}$ & & $155.7, \mathrm{C}$ & & 167.0, C & \\
\hline 3 & $43.0, \mathrm{C}$ & & $42.8, \mathrm{C}$ & & $58.0, \mathrm{C}$ & \\
\hline $4 a$ & $39.9, \mathrm{CH}_{2}$ & $1.50, \mathrm{~m}$ & 36.7, $\mathrm{CH}_{2}$ & $1.23, \mathrm{~m}$ & 29.6, $\mathrm{CH}_{2}$ & $1.42, \mathrm{dd}(13.2,6.0)$ \\
\hline $4 b$ & & $1.36, \mathrm{~m}$ & & $1.74, \mathrm{~m}$ & & $1.55, \mathrm{dd}(12.8,6.0)$ \\
\hline $5 a$ & $25.2, \mathrm{CH}_{2}$ & $1.58, \mathrm{~m}$ & $32.3, \mathrm{CH}_{2}$ & $1.44, \mathrm{~m}$ & $25.9, \mathrm{CH}_{2}$ & $0.90, \mathrm{~m}$ \\
\hline $5 b$ & & $1.24, \mathrm{~m}$ & & $1.55, \mathrm{~m}$ & & $1.80, \mathrm{~m}$ \\
\hline 6 & $37.6, \mathrm{CH}$ & $1.65, \mathrm{~m}$ & $73.7, \mathrm{C}$ & & $46.4, \mathrm{CH}$ & $1.05, \mathrm{~m}$ \\
\hline 7 & $42.2, \mathrm{CH}$ & $2.46, \mathrm{~s}$ & $47.8, \mathrm{CH}$ & 2.44, br s & $42.7, \mathrm{CH}$ & 3.06 , br s \\
\hline $8 a$ & $20.8, \mathrm{CH}_{3}$ & $1.05, \mathrm{~s}$ & $20.8, \mathrm{CH}_{3}$ & $1.06, \mathrm{~s}$ & $64.6, \mathrm{CH}_{2}$ & 3.63, d (11.6) \\
\hline $8 b$ & & & & & & 3.71, d (11.6) \\
\hline 9 & $40.5, \mathrm{CH}$ & $1.46, \mathrm{~m}$ & $36.9, \mathrm{CH}$ & $1.57, \mathrm{~m}$ & $32.9, \mathrm{CH}$ & $1.02, \mathrm{~m}$ \\
\hline 10 & $15.4, \mathrm{CH}_{3}$ & $0.92, \mathrm{~d}(6.8)$ & $16.2, \mathrm{CH}_{3}$ & $0.88, \mathrm{~d}(6.9)$ & $21.1, \mathrm{CH}_{3}$ & $0.78, \mathrm{~d}(6.4)$ \\
\hline 11 & $66.9, \mathrm{CH}_{2}$ & 3.64 , overlap & $16.4, \mathrm{CH}_{3}$ & $0.94, \mathrm{~d}(6.9)$ & $22.1, \mathrm{CH}_{3}$ & $1.06, \mathrm{~d}(6.4)$ \\
\hline $12 \mathrm{a}$ & $103.5, \mathrm{CH}_{2}$ & $4.94, \mathrm{~s}$ & $105, \mathrm{CH}_{2}$ & $4.69, \mathrm{~s}$ & $11.0, \mathrm{CH}_{3}$ & $2.13, \mathrm{~s}$ \\
\hline $12 b$ & & $4.62, \mathrm{~s}$ & & $4.97, \mathrm{~s}$ & & \\
\hline 13 & $58.2, \mathrm{CH}$ & 1.70, br s & $54.7, \mathrm{CH}$ & 1.97 , br s & $60.8, \mathrm{CH}$ & $1.82, \mathrm{~m}$ \\
\hline $14 \mathrm{a}$ & $69.6, \mathrm{CH}$ & $4.02, \mathrm{~d}(5.9)$ & $69.5, \mathrm{CH}$ & $4.07, \mathrm{~d}(6.1)$ & $62.9, \mathrm{CH}_{2}$ & $3.34, \mathrm{dd}(11.0,6.8)$ \\
\hline $14 b$ & & & & & & $3.61, \mathrm{dd}(11.2,6.8)$ \\
\hline 15 & $74.9, \mathrm{CH}$ & 3.65, overlap & $74.8, \mathrm{CH}$ & $3.68, \mathrm{~d}(6.1)$ & 190.0, CH & $10.02, \mathrm{~s}$ \\
\hline
\end{tabular}

${ }^{a}$ Measured in $\mathrm{CDCl}_{3} ;{ }^{b}$ Measured in methanol- $d_{4}$.

Bipolarisorokin B (2): colorless oil; $[\alpha]^{22} \mathrm{D}-100.1$ (c 0.05, MeOH); UV (MeOH) $\lambda_{\max }$ $(\log \varepsilon) 210$ (3.23); ${ }^{1} \mathrm{H}$ NMR (600 MHz, CDCl 3$)$ and $\left.{ }^{13} \mathrm{C} \mathrm{NMR} \mathrm{(150} \mathrm{MHz}, \mathrm{CDCl}_{3}\right)$ data, see Table 1; positive ion HRESIMS $m / z 275.16166[\mathrm{M}+\mathrm{Na}]^{+}$, (calculated for $\mathrm{C}_{15} \mathrm{H}_{24} \mathrm{O}_{3} \mathrm{Na}^{+}$ 275.16177).

Bipolarisorokin C (3): colorless, needle-like crystals $(\mathrm{MeOH}) ; \mathrm{mp} 135-138{ }^{\circ} \mathrm{C} ;[\alpha]^{22} \mathrm{D}-$ 21.8 (c 0.05, MeOH); UV (MeOH) $\lambda_{\max }(\log \varepsilon) 265$ (3.49) nm; ${ }^{1} \mathrm{H}$ NMR (600 MHz, methanol$\left.d_{4}\right)$ and ${ }^{13} \mathrm{C}$ NMR (150 MHz, methanol- $d_{4}$ ) data, see Table 1 ; positive ion HRESIMS $\mathrm{m} / \mathrm{z}$ 253.17971 $[\mathrm{M}+\mathrm{H}]^{+}$(calculated for $\mathrm{C}_{15} \mathrm{H}_{25} \mathrm{O}_{3}{ }^{+} 253.17982$ ).

Bipolarisorokin D (4): colorless oil; $[\alpha]^{25} \mathrm{D}+32.0(c 0.05, \mathrm{MeOH}) ; \mathrm{UV}(\mathrm{MeOH}) \lambda_{\max }(\log$ ع) 255 (3.65); ${ }^{1} \mathrm{H}$ NMR (600 MHz, methanol- $\left.d_{4}\right)$ and ${ }^{13} \mathrm{C}$ NMR (150 MHz, methanol- $\left.d_{4}\right)$ data, see Table 2; positive ion HRESIMS $m / z 275.16153[\mathrm{M}+\mathrm{Na}]^{+}$(calculated for $\mathrm{C}_{15} \mathrm{H}_{24} \mathrm{NaO}_{3}{ }^{+}$ 275.16177). Bipolarisorokin E (5): colorless oil; $[\alpha]^{25} \mathrm{D}-22.7$ (c 0.05, MeOH); UV (MeOH) $\lambda_{\max }$ $(\log \varepsilon) 210$ (3.24); ${ }^{1} \mathrm{H}$ NMR (600 MHz, methanol- $\left.d_{4}\right)$ and ${ }^{13} \mathrm{C}$ NMR (150 MHz, methanol- $\left.d_{4}\right)$ data, see Table 2; positive ion HRESIMS $m / z 221.15529[\mathrm{M}-\mathrm{H}]^{-}$(calculated for $\mathrm{C}_{14} \mathrm{H}_{21} \mathrm{O}_{2}{ }^{-}$ 221.15470).

Bipolarisorokin F (6): white powder; $[\alpha]^{20}{ }_{\mathrm{D}}-3.3($ c $0.04, \mathrm{MeOH}) ; \mathrm{UV}(\mathrm{MeOH}) \lambda_{\max }(\log$ ع) 215 (3.72); ${ }^{1} \mathrm{H}$ NMR (600 MHz, $\left.\mathrm{CDCl}_{3}\right)$ and $\left.{ }^{13} \mathrm{C} \mathrm{NMR} \mathrm{(150} \mathrm{MHz}, \mathrm{CDCl}_{3}\right)$ data, see Table 2; positive ion HRESIMS $m / z 225.18506[\mathrm{M}+\mathrm{H}]^{+}$(calculated for $\mathrm{C}_{14} \mathrm{H}_{25} \mathrm{O}_{2}{ }^{+}$225.18491).

Bipolarisorokin G (7): colorless oil; $[\alpha]^{20} \mathrm{D}+17.2$ (c 0.02, $\left.\mathrm{MeOH}\right) ; \mathrm{UV}(\mathrm{MeOH}) \lambda_{\max }(\log$ ع) 230 (3.21); ${ }^{1} \mathrm{H}$ NMR (600 MHz, $\left.\mathrm{CDCl}_{3}\right)$ and ${ }^{13} \mathrm{C} \mathrm{NMR} \mathrm{(150} \mathrm{MHz,} \mathrm{CDCl}_{3}$ ) data, see Table 3; positive ion HRESIMS $m / z 275.20059[\mathrm{M}+\mathrm{H}]^{+}$(calculated for $\mathrm{C}_{18} \mathrm{H}_{27} \mathrm{O}_{2}{ }^{+}$275.20056). 
Table 2. ${ }^{1} \mathrm{H}(600 \mathrm{MHz})$ and ${ }^{13} \mathrm{C}(150 \mathrm{MHz}) \mathrm{NMR}$ Spectroscopic Data for 4-6.

\begin{tabular}{|c|c|c|c|c|c|c|}
\hline \multirow{2}{*}{ No. } & \multicolumn{2}{|r|}{$4^{b}$} & \multicolumn{2}{|r|}{$5^{b}$} & \multicolumn{2}{|c|}{$6^{a}$} \\
\hline & $\delta_{\mathrm{C}}$, Type & $\delta_{\mathrm{H}}(J$ in $\mathrm{Hz})$ & $\delta_{\mathrm{C}}$, Type & $\delta_{\mathrm{H}}(J$ in $\mathrm{Hz})$ & $\delta_{\mathrm{C}}$, Type & $\delta_{\mathrm{H}}(J$ in $\mathrm{Hz})$ \\
\hline 1 & $140.5, \mathrm{C}$ & & $124.2, \mathrm{CH}$ & 5.56, br s & $212.0, \mathrm{C}$ & \\
\hline 2 & $170.4, \mathrm{C}$ & & $147.2, \mathrm{C}$ & & $50.7, \mathrm{CH}$ & $2.10, \mathrm{~m}$ \\
\hline 3 & $52.0, \mathrm{C}$ & & 47.7, C & & $41.8, \mathrm{C}$ & \\
\hline $4 a$ & $32.4, \mathrm{CH}_{2}$ & $1.38, \mathrm{~m}$ & $35.2, \mathrm{CH}_{2}$ & $1.34, \mathrm{~m}$ & $36.1, \mathrm{CH}_{2}$ & $1.44, \mathrm{~m}$ \\
\hline $4 b$ & & $1.71, \mathrm{~m}$ & & $1.41, \mathrm{dd}(12.5,5.2)$ & & $1.66, \mathrm{dd}(13.7,5.7)$ \\
\hline $5 a$ & $32.8, \mathrm{CH}_{2}$ & $1.25, \mathrm{~m}$ & 26.0, $\mathrm{CH}_{2}$ & $1.56, \mathrm{~m}$ & 26.0, $\mathrm{CH}_{2}$ & $1.80, \mathrm{~m}$ \\
\hline $5 b$ & & $1.61, \mathrm{~m}$ & & & & $0.87, \mathrm{~m}$ \\
\hline 6 & $73.5, \mathrm{C}$ & & $45.2, \mathrm{CH}$ & $2.03, \mathrm{~m}$ & $50.1, \mathrm{CH}$ & $1.33, \mathrm{~m}$ \\
\hline 7 & $47.9, \mathrm{CH}$ & 3.16 , br s & $45.3, \mathrm{CH}$ & 2.74, br s & $51.3, \mathrm{CH}$ & 2.70, brs \\
\hline 8 & $18.7, \mathrm{CH}_{3}$ & $1.07, \mathrm{~s}$ & $18.9, \mathrm{CH}_{3}$ & $0.99, \mathrm{~s}$ & $22.1, \mathrm{CH}_{3}$ & $1.09, \mathrm{~s}$ \\
\hline 9 & $37.2, \mathrm{CH}$ & $1.28, \mathrm{~m}$ & $150.3, \mathrm{C}$ & & $29.9, \mathrm{CH}$ & $1.55, \mathrm{~m}$ \\
\hline 10 & $17.1, \mathrm{CH}_{3}$ & $1.02, \mathrm{~d}(6.6)$ & $109.2, \mathrm{CH}_{2}$ & $4.69, \mathrm{~d}(5.1)$ & $20.3, \mathrm{CH}_{3}$ & $1.03, \mathrm{~d}(6.5)$ \\
\hline 11 & $16.4, \mathrm{CH}_{3}$ & $0.80, \mathrm{~d}(6.6)$ & 22.7, $\mathrm{CH}_{3}$ & $1.74, \mathrm{~s}$ & $21.4, \mathrm{CH}_{3}$ & $0.86, \mathrm{~d}(6.5)$ \\
\hline 12 & $11.3, \mathrm{CH}_{2}$ & $2.06, \mathrm{~s}$ & $59.8, \mathrm{CH}_{2}$ & $4.06, \mathrm{~m}$ & $6.3, \mathrm{CH}_{3}$ & $0.96, \mathrm{~d}(7.2)$ \\
\hline 13 & $55.3, \mathrm{CH}$ & $2.43, \mathrm{dd}(9.1,5.4)$ & $64.3, \mathrm{CH}$ & $1.64, \mathrm{dd}(9.6,4.9)$ & $54.9, \mathrm{C}$ & $1.72, \mathrm{dd}(7.9,5.0)$ \\
\hline $14 \mathrm{a}$ & 62.3, $\mathrm{CH}_{2}$ & $3.19, \mathrm{dd}(10.5,9.1)$ & $62.5, \mathrm{CH}_{2}$ & $3.38, \mathrm{~m}$ & 62.0, $\mathrm{CH}_{2}$ & $3.85, \mathrm{dd}(10.7,5.0)$ \\
\hline $14 \mathrm{~b}$ & & 3.61 , dd $(10.5,5.4)$ & & 3.65 , dd $(10.5,5.0)$ & & $3.50, \mathrm{dd}(10.7,7.9)$ \\
\hline 15 & 189.7, CH & $9.97, \mathrm{~s}$ & & & & \\
\hline
\end{tabular}

${ }^{a}$ Measured in $\mathrm{CDCl}_{3} ;{ }^{b}$ Measured in methanol- $d_{4}$.

Table 3. ${ }^{1} \mathrm{H}(600 \mathrm{MHz})$ and ${ }^{13} \mathrm{C}(150 \mathrm{MHz})$ NMR Spectroscopic Data for 7-9.

\begin{tabular}{|c|c|c|c|c|c|c|}
\hline \multirow{2}{*}{ No. } & \multicolumn{2}{|r|}{$7^{a}$} & \multicolumn{2}{|c|}{$8^{b}$} & \multicolumn{2}{|r|}{$9^{b}$} \\
\hline & $\delta_{\mathrm{C}}$, Type & $\delta_{\mathrm{H}}(J$ in $\mathrm{Hz})$ & $\delta_{\mathrm{C}}$, Type & $\delta_{\mathbf{H}}(J$ in $\mathbf{H z})$ & $\delta_{\mathrm{C}}$, Тype & $\delta_{\mathrm{H}}(J$ in $\mathrm{Hz})$ \\
\hline 1 & $137.5, \mathrm{C}$ & & $137.4, \mathrm{C}$ & & $57.5, \mathrm{CH}$ & 2.54, br s \\
\hline 2 & 165.3, C & & 165.3, C & & 163.7, C & \\
\hline 3 & $52.6, \mathrm{C}$ & & $52.5, \mathrm{C}$ & & $41.8, \mathrm{C}$ & \\
\hline $4 a$ & $33.7, \mathrm{CH}_{2}$ & $1.41, \mathrm{dd}(13.4,5.9)$ & 33.6, $\mathrm{CH}_{2}$ & $1.40, \mathrm{dd}(13.3,5.9)$ & $53.2, \mathrm{CH}_{2}$ & $1.66, \mathrm{dd}(13.2,10.4)$ \\
\hline $4 b$ & & $1.50, \mathrm{dd}(13.4,6.4)$ & & $1.48, \mathrm{dd}(13.3,6.5)$ & & $2.10, \mathrm{dd}(13.2,10.4)$ \\
\hline $5 a$ & $25.2, \mathrm{CH}_{2}$ & $0.91, \mathrm{~m}$ & $25.2, \mathrm{CH}_{2}$ & $0.90, \mathrm{~m}$ & $67.0, \mathrm{CH}$ & $3.84, \mathrm{~m}$ \\
\hline $5 b$ & & $1.80, \mathrm{~m}$ & & $1.78, \mathrm{~m}$ & & \\
\hline $6 a$ & $44.3, \mathrm{CH}$ & $1.06, \mathrm{~m}$ & $44.2, \mathrm{CH}$ & $1.06, \mathrm{~m}$ & $47.2, \mathrm{CH}_{2}$ & $1.21, \mathrm{~m}$ \\
\hline $6 b$ & & & & & & $1.98, \mathrm{~m}$ \\
\hline 7 & $44.7, \mathrm{CH}$ & 3.06, br s & $44.5, \mathrm{CH}$ & 3.04, br s & $32.2, \mathrm{C}$ & \\
\hline 8 & $19.7, \mathrm{CH}_{3}$ & $0.97, \mathrm{~s}$ & $19.6, \mathrm{CH}_{3}$ & $0.96, \mathrm{~s}$ & 28.7, $\mathrm{CH}_{3}$ & $0.99, \mathrm{~s}$ \\
\hline 9 & $31.6, \mathrm{CH}$ & $1.03, \mathrm{~m}$ & $31.6, \mathrm{CH}$ & $1.03, \mathrm{~m}$ & $55.0, \mathrm{CH}$ & 2.02, br s \\
\hline 10 & 21.7, $\mathrm{CH}_{3}$ & $1.06, \mathrm{~d}(5.9)$ & 21.7, $\mathrm{CH}_{3}$ & $1.04, \mathrm{~d}(5.8)$ & $30.3, \mathrm{CH}_{3}$ & $1.09, \mathrm{~s}$ \\
\hline 11 & $20.8, \mathrm{CH}_{3}$ & 0.77 , d (5.9) & $20.8, \mathrm{CH}_{3}$ & $0.76, \mathrm{~d}(5.8)$ & $31.7, \mathrm{CH}_{3}$ & $0.95, \mathrm{~s}$ \\
\hline $12 \mathrm{a}$ & $11.0, \mathrm{CH}_{3}$ & $2.06, \mathrm{~s}$ & $10.9, \mathrm{CH}_{3}$ & $2.04, \mathrm{~s}$ & $103.9, \mathrm{CH}_{2}$ & 4.75 , br s \\
\hline $12 b$ & & & & & & 4.97, br s \\
\hline 13 & $63.6, \mathrm{CH}$ & $2.22, \mathrm{~d}(9.6)$ & $63.4, \mathrm{CH}$ & $2.23, \mathrm{~d}(9.8)$ & $53.2, \mathrm{CH}$ & $2.01, \mathrm{br} \mathrm{s}$ \\
\hline 14 & $147.9, \mathrm{CH}$ & $6.55, \mathrm{dd}(15.9,9.6)$ & $151.5, \mathrm{CH}$ & 6.80, dd $(15.4,9.9)$ & $70.5, \mathrm{CH}$ & $4.13, \mathrm{~d}(6.2)$ \\
\hline 15 & $188.1, \mathrm{CH}$ & $10.08, \mathrm{~s}$ & $188.1, \mathrm{CH}$ & $10.05, \mathrm{~s}$ & $74.9, \mathrm{CH}$ & $3.59, \mathrm{~d}(6.2)$ \\
\hline 16 & $132.2, \mathrm{CH}$ & $6.08, \mathrm{~d}(15.9)$ & 122.1, $\mathrm{CH}$ & $5.81, \mathrm{~d}(15.5)$ & & \\
\hline 17 & $198.6, \mathrm{C}$ & & 171.1, C & & & \\
\hline 18 & $27.5, \mathrm{CH}_{3}$ & $2.20, \mathrm{~s}$ & & & & \\
\hline
\end{tabular}

${ }^{a}$ Measured in $\mathrm{CDCl}_{3} ;{ }^{b}$ Measured in methanol- $d_{4}$.

Bipolarisorokin $H(8)$ : colorless oil; $[\alpha]^{25} \mathrm{D}-136.9$ (c 0.05, MeOH); UV (MeOH) $\lambda_{\max }$ $(\log \varepsilon) 225$ (3.93); ${ }^{1} \mathrm{H}$ NMR (600 MHz, CDCl 3 ) and $\left.{ }^{13} \mathrm{C} \mathrm{NMR} \mathrm{(150} \mathrm{MHz,} \mathrm{CDCl}_{3}\right)$ data, see Table 3; positive ion HRESIMS $m / z 277.17984[\mathrm{M}+\mathrm{H}]^{+}$(calculated for $\mathrm{C}_{17} \mathrm{H}_{25} \mathrm{O}_{3}{ }^{+}$277.17982).

Bipolarisorokin I (9): colorless crystals; mp 191-194 ${ }^{\circ} \mathrm{C} ;[\alpha]^{22} \mathrm{D}+8.8(c 0.05, \mathrm{MeOH}) ; \mathrm{UV}$ $(\mathrm{MeOH}) \lambda_{\max }(\log \varepsilon) 210$ (3.46); ${ }^{1} \mathrm{H}$ NMR $\left(600 \mathrm{MHz}\right.$, methanol- $\left.d_{4}\right)$ and ${ }^{13} \mathrm{C} \mathrm{NMR}(150 \mathrm{MHz}$, methanol- $d_{4}$ ) data, see Table 3; positive ion HRESIMS $m / z 251.16621$ [M-H] ${ }^{-}$, (calculated for $\left.\mathrm{C}_{21} \mathrm{H}_{23} \mathrm{O}_{3}-251.16527\right)$. 
Bipolarithone A (10): colorless oil; $[\alpha]^{23} \mathrm{D}+136.0$ (c 0.05, MeOH); UV (MeOH) $\lambda_{\max }(\log$ ع) 245 (3.30); ${ }^{1} \mathrm{H}$ NMR (600 MHz, $\left.\mathrm{CDCl}_{3}\right)$ and ${ }^{13} \mathrm{C} \mathrm{NMR}\left(150 \mathrm{MHz}, \mathrm{CDCl}_{3}\right)$ data, see Table 4; positive ion HRESIMS m/z $349.09143[\mathrm{M}+\mathrm{H}]^{+}$, (calculated for $\mathrm{C}_{17} \mathrm{H}_{17} \mathrm{O}_{8}{ }^{+}$349.09179).

Table 4. ${ }^{1} \mathrm{H}(600 \mathrm{MHz})$ and ${ }^{13} \mathrm{C}(150 \mathrm{MHz})$ NMR Spectroscopic Data for 10 and 11.

\begin{tabular}{|c|c|c|c|c|}
\hline \multirow{2}{*}{ No. } & \multicolumn{2}{|r|}{$10^{a}$} & \multicolumn{2}{|r|}{$11^{a}$} \\
\hline & $\delta_{\mathrm{C}}$, Type & $\delta_{\mathrm{H}}(J$ in $\mathrm{Hz})$ & $\delta_{\mathrm{C}}$, Type & $\delta_{\mathrm{H}}(J$ in $\mathrm{Hz})$ \\
\hline 1 & $161.1, \mathrm{C}$ & & $161.1, \mathrm{C}$ & \\
\hline 2 & 113.7, CH & $6.68, \mathrm{~s}$ & $113.7, \mathrm{CH}$ & $6.69, \mathrm{~s}$ \\
\hline 3 & $147.7, \mathrm{C}$ & & $147.8, \mathrm{C}$ & \\
\hline 4 & 108.1, CH & $6.75, \mathrm{~s}$ & 108.1, CH & $6.76, \mathrm{~s}$ \\
\hline $4 a$ & $157.4, \mathrm{C}$ & & $157.4, \mathrm{C}$ & \\
\hline 5 & $78.2, \mathrm{CH}$ & 5.73, ddd $(6.6,4.4,3.9)$ & $78.6, \mathrm{CH}$ & 5.62 , ddd $(8.4,3.8,1.7)$ \\
\hline $6 a$ & $37.7, \mathrm{CH}_{2}$ & 3.01, dd $(16.2,4.4)$ & 39.3, $\mathrm{CH}_{2}$ & 3.10, dd $(16.3,8.4)$ \\
\hline $6 b$ & & $2.85, \mathrm{dd}(16.2,6.6)$ & & 2.99, dd $(16.3,3.8)$ \\
\hline 7 & $170.0, \mathrm{C}$ & & $170.2, \mathrm{C}$ & \\
\hline 8 & $79.4, \mathrm{CH}$ & $5.64, \mathrm{~d}(3.9)$ & $79.8, \mathrm{CH}$ & $5.63, \mathrm{~d}(1.7)$ \\
\hline $8 a$ & $114.7, \mathrm{C}$ & & $114.6, \mathrm{C}$ & \\
\hline 9 & 178.3, C & & $178.2, \mathrm{C}$ & \\
\hline $9 a$ & 109.0, C & & 109.0, C & \\
\hline $10 a$ & $167.7, \mathrm{C}$ & & $167.4, \mathrm{C}$ & \\
\hline $1^{\prime}$ & $22.5, \mathrm{CH}_{3}$ & $2.41, \mathrm{~s}$ & $22.5, \mathrm{CH}_{3}$ & $2.42, \mathrm{~s}$ \\
\hline $2^{\prime}$ & $169.5, \mathrm{C}$ & & $170.1, \mathrm{C}$ & \\
\hline $3^{\prime}$ & $52.4, \mathrm{CH}_{3}$ & $3.73, \mathrm{~s}$ & $52.5, \mathrm{CH}_{3}$ & $3.78, \mathrm{~s}$ \\
\hline $4^{\prime}$ & $53.0, \mathrm{CH}_{3}$ & $3.81, \mathrm{~s}$ & $53.1, \mathrm{CH}_{3}$ & $3.83, \mathrm{~s}$ \\
\hline $1-\mathrm{OH}$ & & $12.06, \mathrm{~s}$ & & $12.01, \mathrm{~s}$ \\
\hline
\end{tabular}

${ }^{a}$ Measured in $\mathrm{CDCl}_{3} ;{ }^{b}$ Measured in methanol- $d_{4}$.

Bipolarithone B (11): colorless oil; $[\alpha]^{23} \mathrm{D}-24.2$ (c 0.05, MeOH); UV (MeOH) $\lambda_{\max }(\log$ ع) 245 (3.30); ${ }^{1} \mathrm{H}$ NMR (600 MHz, $\left.\mathrm{CDCl}_{3}\right)$ and ${ }^{13} \mathrm{C} \mathrm{NMR} \mathrm{(150} \mathrm{MHz,} \mathrm{CDCl} 3$ ) data, see Table 4; positive ion HRESIMS $m / z 349.09157[\mathrm{M}+\mathrm{H}]^{+}$, (calculated for $\mathrm{C}_{17} \mathrm{H}_{17} \mathrm{O}_{8}{ }^{+}$349.09179).

Bipolarithone C (12): colorless oil; $[\alpha]^{25} \mathrm{D}+52.9(c 0.5, \mathrm{MeOH}) ; \mathrm{UV}(\mathrm{MeOH}) \lambda_{\max }(\log \varepsilon)$ 245 (4.06); ${ }^{1} \mathrm{H}$ NMR (600 MHz, CDCl $)$ and ${ }^{13} \mathrm{C} \mathrm{NMR} \mathrm{(150} \mathrm{MHz,} \mathrm{CDCl} 3$ ) data, see Table 5; positive ion HRESIMS $m / z 541.24310[\mathrm{M}+\mathrm{H}]^{+}$, (calculated for $\mathrm{C}_{30} \mathrm{H}_{37} \mathrm{O}_{9}{ }^{+}$541.24321).

Bipolarithone D (13): colorless oil; $[\alpha]^{25} \mathrm{D}+10.2(c 0.5, \mathrm{MeOH}) ; \mathrm{UV}(\mathrm{MeOH}) \lambda_{\max }(\log \varepsilon)$ 245 (3.88); ${ }^{1} \mathrm{H}$ NMR (600 $\left.\mathrm{MHz} \mathrm{CDCl}_{3}\right)$ and $\left.{ }^{13} \mathrm{C} \mathrm{NMR} \mathrm{(150} \mathrm{MHz,} \mathrm{CDCl}_{3}\right)$ data, see Table 5; positive ion HRESIMS $m / z 541.24316[\mathrm{M}+\mathrm{H}]^{+}$, (calculated for $\mathrm{C}_{30} \mathrm{H}_{37} \mathrm{O}_{9}{ }^{+}$541.24321).

Crystal data for $\mathrm{Cu} \_1 \_0 m: \mathrm{C}_{15} \mathrm{H}_{24} \mathrm{O}_{3}, M=252.34$, $\mathrm{a}=9.7038(6) \AA$, $\mathrm{b}=13.7866(8)$ $\AA, \mathrm{c}=16.6333(10) \AA, \alpha=95.329(3)^{\circ}, \beta=104.898(2)^{\circ}, \gamma=102.525(3)^{\circ}, V=2073.0(2) \AA^{3}$, $T=100(2) \mathrm{K}$, space group $\mathrm{P} 1, \mathrm{Z}=6, \mu(\mathrm{Cu} \mathrm{K} \alpha)=1.54178 \mathrm{~mm}^{-1}, F(000)=828,82979$ reflections measured, 16831 independent reflections $\left(R_{\text {int }}=0.0695\right)$. The final $R_{1}$ values were $0.0437(I>2 \sigma(I))$. The final $w R\left(F^{2}\right)$ values were $0.1047(I>2 \sigma(I))$. The final $R_{1}$ values were 0.0531 (all data). The final $w R\left(F^{2}\right)$ values were 0.1143 (all data). The goodness of fit on $F^{2}$ was 1.039. Flack parameter $=-0.10(7)$. CCDC: 2124305 . Available online: https: / / www.ccdc.cam.ac.uk (accessed on 11 December 2021).

Crystal data for $\mathrm{Cu}$ _3_0m: $\mathrm{C}_{15} \mathrm{H}_{24} \mathrm{O}_{3}, M=252.34$, $\mathrm{a}=7.0044(5) \AA$, $\mathrm{b}=10.1468(8)$ $\AA, \mathrm{c}=20.1433(14) \AA, \alpha=90.00^{\circ}, \beta=90.00^{\circ}, \gamma=90.00^{\circ}, V=1431.63(18) \AA^{3}, T=295(2)$ $\mathrm{K}$, space group P 2121 21, with $Z=4, \mu(\mathrm{Cu} \mathrm{K} \alpha)=1.54178 \mathrm{~mm}^{-1}, F(000)=552,6263$ reflections measured, 2527 independent reflections $\left(R_{\text {int }}=0.0500\right)$. The final $R_{1}$ values were $0.0519(I>2 \sigma(I))$. The final $w R\left(F^{2}\right)$ values were $0.1538(I>2 \sigma(I))$. The final $R_{1}$ values were 0.0719 (all data). The final $w R\left(F^{2}\right)$ values were 0.2087 (all data). The goodness of fit on $F^{2}$ was 1.117. Flack parameter $=-0.40(17)$. CCDC: 2124306 . Available online: https:/ / www.ccdc.cam.ac.uk (accessed on 11 December 2021). 
Table 5. ${ }^{1} \mathrm{H}(600 \mathrm{MHz})$ and ${ }^{13} \mathrm{C}(150 \mathrm{MHz})$ NMR Spectroscopic Data for 12 and 13.

\begin{tabular}{|c|c|c|c|c|}
\hline \multirow{2}{*}{ No. } & \multicolumn{2}{|r|}{$12^{a}$} & \multicolumn{2}{|r|}{$13^{a}$} \\
\hline & $\delta_{\mathrm{C}}$, Type & $\delta_{\mathrm{H}}(J$ in $\mathrm{Hz})$ & $\delta_{\mathrm{C}}$, Type & $\delta_{\mathrm{H}}(J$ in $\mathrm{Hz})$ \\
\hline 1 & 221.6, C & & $221.4, \mathrm{C}$ & \\
\hline 2 & $50.6, \mathrm{CH}$ & $2.16, \mathrm{~m}$ & $50.6, \mathrm{CH}$ & $2.13, \mathrm{~m}$ \\
\hline 3 & $42.1, \mathrm{C}$ & & $42.0, \mathrm{C}$ & \\
\hline $4 a$ & 36.0, $\mathrm{CH} 2$ & $1.45, \mathrm{~m}$ & $36.1, \mathrm{CH}_{2}$ & $1.47, \mathrm{~m}$ \\
\hline $4 b$ & & $1.66, \mathrm{~m}$ & & $1.67, \mathrm{~m}$ \\
\hline $5 a$ & 26.0, $\mathrm{CH} 2$ & $0.84, \mathrm{~m}$ & $26.0, \mathrm{CH}_{2}$ & $0.83, \mathrm{~m}$ \\
\hline $5 b$ & & $1.78, \mathrm{~m}$ & & $1.79, \mathrm{~m}$ \\
\hline 6 & $50.2, \mathrm{CH}$ & $1.29, \mathrm{~m}$ & $50.1, \mathrm{CH}$ & $1.28, \mathrm{~m}$ \\
\hline 7 & $51.5, \mathrm{CH}$ & $2.56, \mathrm{br} \mathrm{s}$ & $51.5, \mathrm{CH}$ & $2.62, \mathrm{br} \mathrm{s}$ \\
\hline 8 & $22.1, \mathrm{CH} 3$ & $1.08, \mathrm{~s}$ & $22.1, \mathrm{CH}_{3}$ & $1.09, \mathrm{~s}$ \\
\hline 9 & $29.9, \mathrm{CH}$ & $1.41, \mathrm{~m}$ & $30.0, \mathrm{CH}$ & $1.43, \mathrm{~m}$ \\
\hline 10 & 20.4, CH3 & $0.77, \mathrm{~d}(6.6)$ & 20.4, $\mathrm{CH}_{3}$ & $0.78, d(6.7)$ \\
\hline 11 & 21.3, $\mathrm{CH} 3$ & $0.89, \mathrm{~d}(6.4)$ & 21.3, $\mathrm{CH}_{3}$ & $0.92, \mathrm{~d}(6.5)$ \\
\hline 12 & $6.5, \mathrm{CH} 3$ & $0.95, \mathrm{~d}(7.2)$ & $6.5, \mathrm{CH}_{3}$ & $0.96, \mathrm{~d}(7.2)$ \\
\hline 13 & $51.6, \mathrm{CH}$ & $1.90, \mathrm{~m}$ & $51.6, \mathrm{CH}$ & $1.94, \mathrm{~m}$ \\
\hline $14 \mathrm{a}$ & $65.3, \mathrm{CH} 2$ & $4.05, \mathrm{dd}(11.3,5.1)$ & $65.5, \mathrm{CH}_{2}$ & $4.04, \mathrm{dd}(11.3,5.2)$ \\
\hline $14 \mathrm{~b}$ & & 4.35, dd $(11.3,5.1)$ & & 4.37, dd $(11.3,5.2)$ \\
\hline $1^{\prime}$ & 161.1, C & & 161.1, C & \\
\hline $2^{\prime}$ & $113.6, \mathrm{CH}$ & $6.67, \mathrm{~s}$ & 113.7, $\mathrm{CH}$ & $6.68, \mathrm{~s}$ \\
\hline $3^{\prime}$ & $147.6, \mathrm{C}$ & & $147.8, \mathrm{C}$ & \\
\hline $4^{\prime}$ & $108.1, \mathrm{CH}$ & $6.75, \mathrm{~s}$ & $108.1, \mathrm{CH}$ & $6.75, \mathrm{~s}$ \\
\hline $4 a^{\prime}$ & 157.3, C & & $157.4, \mathrm{C}$ & \\
\hline $5^{\prime}$ & $78.2, \mathrm{CH}$ & 5.67 , ddd $(6.4,4.3,3.9)$ & $78.5, \mathrm{CH}$ & 5.59, ddd $(8.2,3.9,1.8)$ \\
\hline $6^{\prime} \mathrm{a}$ & $37.7, \mathrm{CH} 2$ & $2.99, \mathrm{dd}(16.1,4.3)$ & $39.2, \mathrm{CH}_{2}$ & 3.07, dd $(16.3,8.2)$ \\
\hline $6^{\prime} b$ & & $2.84, \mathrm{dd}(16.1,6.4)$ & & 2.99, dd $(16.3,3.9)$ \\
\hline $7^{\prime}$ & $169.5, \mathrm{C}$ & & $170.0, \mathrm{C}$ & \\
\hline $8^{\prime}$ & $79.5, \mathrm{CH}$ & $5.59, \mathrm{~d}(3.9)$ & $79.9, \mathrm{CH}$ & $5.58, \mathrm{~d}(1.8)$ \\
\hline $8 a^{\prime}$ & $114.5, \mathrm{C}$ & & $114.5, \mathrm{C}$ & \\
\hline $9^{\prime}$ & $178.2, \mathrm{C}$ & & $178.2, \mathrm{C}$ & \\
\hline $9 a^{\prime}$ & 109.0, C & & 109.0, C & \\
\hline $10 a^{\prime}$ & 167.7, C & & 167.3, C & \\
\hline $1^{\prime \prime}$ & $22.5, \mathrm{CH} 3$ & $2.40, \mathrm{~s}$ & $22.5, \mathrm{CH}_{3}$ & $2.41, \mathrm{~s}$ \\
\hline $2^{\prime \prime}$ & $169.4, \mathrm{C}$ & & $169.7, \mathrm{C}$ & \\
\hline $3^{\prime \prime}$ & $52.4, \mathrm{CH} 3$ & $3.72, \mathrm{~s}$ & $52.5, \mathrm{CH}_{3}$ & $3.72, \mathrm{~s}$ \\
\hline $1^{\prime}-\mathrm{OH}$ & & $12.06, \mathrm{~s}$ & & $12.06, \mathrm{~s}$ \\
\hline
\end{tabular}

${ }^{a}$ Measured in $\mathrm{CDCl}_{3} ;{ }^{b}$ Measured in methanol- $d_{4}$.

Crystal data for $\mathrm{Cu}$ _9_0m: $\mathrm{C}_{15} \mathrm{H}_{24} \mathrm{O}_{3}, M=252.34, \mathrm{a}=6.8634(2) \AA, \mathrm{b}=15.0872(4) \AA$ $\mathrm{c}=13.5156(3) \AA, \alpha=90.00^{\circ}, \beta=90.4010(10)^{\circ}, \gamma=90.00^{\circ}, V=1399.50(6) \AA^{3}, \mathrm{~T}=295(2)$ $\mathrm{K}$, space group P 121 , with $\mathrm{Z}=4, \mu(\mathrm{Cu} \mathrm{K} \alpha)=1.54178 \mathrm{~mm}^{-1}, F(000)=552,32232$ reflections measured, 5982 independent reflections $\left(R_{\text {int }}=0.0279\right)$. The final $R_{1}$ values were $0.0300(I>2 \sigma(I))$. The final $w R\left(F^{2}\right)$ values were $0.0808(I>2 \sigma(I))$. The final $R_{1}$ values were 0.0304 (all data). The final $w R\left(F^{2}\right)$ values were 0.0812 (all data). The goodness of fit on $F^{2}$ was 1.057. Flack parameter $=-0.01(3)$. CCDC: 2124307. Available online: https:/ / www.ccdc.cam.ac.uk (accessed on 11 December 2021).

Crystal data for $\mathrm{Cu} \_17 \_0 \mathrm{~m}: \mathrm{C}_{14} \mathrm{H}_{24} \mathrm{O}_{2}, M=224.33, \mathrm{a}=13.6388(2) \AA, \mathrm{b}=13.6388(2)$ $\AA, \mathrm{c}=13.0174(2) \AA, \alpha=90.00^{\circ}, \beta=90.00^{\circ}, \gamma=90.00^{\circ}, V=2097.04(7) \AA^{3}, T=296(2) \mathrm{K}$, space group P 3121 , with $\mathrm{Z}=6, \mu(\mathrm{Cu} \mathrm{K} \alpha)=1.54178 \mathrm{~mm}^{-1}, F(000)=744,39026$ reflections measured, 3033 independent reflections $\left(R_{\text {int }}=0.0459\right)$. The final $R_{1}$ values were 0.0353 $(I>2 \sigma(I))$. The final $w R\left(F^{2}\right)$ values were $0.0988(I>2 \sigma(I))$. The final $R_{1}$ values were 0.0366 (all data). The final $w R\left(F^{2}\right)$ values were 0.1003 (all data). The goodness of fit on $F^{2}$ was 1.047. Flack parameter $=0.01(5)$. CCDC: 2126101 . Available online: https: $/ /$ www.ccdc.cam.ac.uk (accessed on 11 December 2021).

Crystal data for $\mathrm{Cu} \_18 \_0 \mathrm{~m}: \mathrm{C}_{15} \mathrm{H}_{26} \mathrm{O}_{2}, M=238.36, \mathrm{a}=13.1977(2) \AA, \mathrm{b}=13.1977(2)$ $\AA, \mathrm{c}=8.49040(10) \AA, \alpha=90.00^{\circ}, \beta=90.00^{\circ}, \gamma=90.00^{\circ}, V=1478.85(5) \AA^{3}, T=297(2) \mathrm{K}$, 
space group $\mathrm{P} 43$, with $\mathrm{Z}=4, \mu(\mathrm{Cu} \mathrm{K} \alpha)=1.54178 \mathrm{~mm}^{-1}, F(000)=528,14568$ reflections measured, 3063 independent reflections $\left(R_{\text {int }}=0.0269\right)$. The final $R_{1}$ values were 0.0534 $(I>2 \sigma(I))$. The final $w R\left(F^{2}\right)$ values were $0.1525(I>2 \sigma(I))$. The final $R_{1}$ values were 0.0541 (all data). The final $w R\left(F^{2}\right)$ values were 0.1539 (all data). The goodness of fit on $F^{2}$ was 1.051. Flack parameter $=0.12(7)$. CCDC: 2126105 . Available online: https: $/ /$ www.ccdc.cam.ac.uk (accessed on 11 December 2021).

\subsection{ECD Calculations}

The ECD calculations were carried out using the Gaussian 16 software package [26]. Systematic conformational analyses were performed via SYBYL-X 2.1 using the MMFF94 molecular mechanics force field calculation with $10 \mathrm{kcal} / \mathrm{mol}$ of cutoff energy $[27,28]$. The optimization and frequency of conformers were calculated on the B3LYP/6-31G(d) level in the Gaussian 09 program package. The ECD (TDDFT) calculations were performed on the B3LYP/6-311G(d) level of theory with an IEFPCM solvent model (MeOH). The ECD curves were simulated in SpecDis V1.71 using a Gaussian function [29]. The calculated ECD data of all conformers were Boltzmann averaged by Gibbs free energy.

\subsection{NMR Calculations}

All the optimized conformers in an energy window of $5 \mathrm{kcal} / \mathrm{mol}$ (with no imaginary frequency) were subjected to gauge-independent atomic orbital (GIAO) calculations of their ${ }^{13} \mathrm{C}$ NMR chemical shifts, using density functional theory (DFT) at the mPW1PW91/6$311+\mathrm{G}(\mathrm{d}, \mathrm{p})$ level with the PCM model. The calculated NMR data of these conformers were averaged according to the Boltzmann distribution theory and their relative Gibbs free energy. The ${ }^{13} \mathrm{C}$ NMR chemical shifts for TMS were also calculated by the same procedures and used as the reference. After the calculation, the experimental and calculated data were evaluated by the improved probability $\mathrm{DP}^{+}$method [30].

\subsection{Antibacterial Activity Assay}

The bacterium P. syringae pv. actinidiae was donated by Dr. He Yan of Northwest A\&F University, China. A sample of each culture was then diluted 1000-fold in fresh Luria-Bertani (LB) (Beijing Solarbio Science \& Technology. Co. Ltd., Beijing, China) and incubated with shaking $(160 \mathrm{rpm})$ at $27^{\circ} \mathrm{C}$ for $10 \mathrm{~h}$. The resultant mid-log phase cultures were diluted to a concentration of $5 \times 10^{5} \mathrm{CFU} / \mathrm{mL}$, then $160 \mu \mathrm{L}$ was added to each well of the compound-containing plates. Subsequently, 1:1 serial dilutions with sterile PBS of each compound were performed, giving a final compound concentration range from 4 to256 $\mathrm{\mu g} / \mathrm{mL}$. The minimum inhibitory concentration (MIC, with an inhibition rate of $\geq 90 \%$ ) was determined by using photometry at $\mathrm{OD}_{600} \mathrm{~nm}$ after $24 \mathrm{~h}$. Streptomycin was used as the positive control.

\subsection{Anti-Phytopathogens Assay}

Four phytopathogens (Phytophthora infestane, Alternaria solani, Rhizoctonia solani, and Fusarium oxysporum) were cultured in PDA with micro glass beads at $27^{\circ} \mathrm{C}$ for a week, as well as shaking (160 rpm). Ninety microliters of PDA, together with a $10 \mu \mathrm{L}$ volume of an aqueous test sample solution, was added into each well of the 96-well plate. The test solutions contained different concentrations of the sample being tested. Then, agar plugs $\left(1 \mathrm{~mm}^{3}\right)$ with fresh phytopathogens were inoculated into each well. Subsequently, a two-fold serial dilution in the microplate wells was performed over a concentration range of 4 to $256 \mu \mathrm{g} / \mathrm{mL}$. Plates were covered and incubated at $27^{\circ} \mathrm{C}$ for $24 \mathrm{~h}$. Finally, the minimum inhibitory concentration was determined by observing the plates, with no growth in the well taken as that value. Hygromycin B was used as the positive control.

\section{Results and Discussion}

Bipolarisorokin A (1) was isolated as colorless crystals. Its molecular formula of $\mathrm{C}_{15} \mathrm{H}_{24} \mathrm{O}_{3}$ was determined on the basis of the HR-ESIMS data (measured at $\mathrm{m} / \mathrm{z} 251.16624$ 
$[\mathrm{M}-\mathrm{H}]^{-}$, calculated for $\mathrm{C}_{15} \mathrm{H}_{23} \mathrm{O}_{3}{ }^{-}$251.16527), corresponding to four degrees of unsaturation. The ${ }^{1} \mathrm{H}$ and ${ }^{13} \mathrm{C}$ NMR spectra, in association with the HSQC spectrum, revealed two methyls, four methenes, seven methines, and two quaternary carbons (Table 1). Of them, signals at $\delta_{\mathrm{C}} 66.9(\mathrm{t}, \mathrm{C}-11), 69.6(\mathrm{~d}, \mathrm{C}-14)$, and $74.9(\mathrm{~d}, \mathrm{C}-15)$ were identified as the oxygenated methylene and methines. Two olefinic carbons at $\delta_{\mathrm{C}} 156.8(\mathrm{~s}, \mathrm{C}-2)$ and $103.5(\mathrm{t}$, C-12) corresponded to a double bond, which suggested that 1 possessed a tricyclic system. Considering the 15 carbons in $\mathbf{1}$, as well as those isolates from the same source, compound 1 was suggested to be a tricyclic sesquiterpenoid. In the ${ }^{1} \mathrm{H}-{ }^{1} \mathrm{H}$ COSY spectrum, a fragment was revealed, as shown with bold lines in Figure 2. The HMBC correlations from to $\delta_{\mathrm{H}}$ $4.94(\mathrm{H}, \mathrm{s}, \mathrm{H}-12 \mathrm{a})$ and $4.62(\mathrm{H}, \mathrm{s}, \mathrm{H}-12 \mathrm{~b})$, to $\delta_{\mathrm{C}} 156.8(\mathrm{~s}, \mathrm{C}-2), 54.3(\mathrm{~d}, \mathrm{C}-1)$ and $43.0(\mathrm{~s}, \mathrm{C}-3)$, established the connections between $\mathrm{C}-12, \mathrm{C}-2$, and $\mathrm{C}-1$. Further analyses of ${ }^{1} \mathrm{H}-{ }^{1} \mathrm{H}$ COSY, as well as HMBC correlations from $\delta_{\mathrm{H}} 0.92(3 \mathrm{H}, \mathrm{d}, J=6.8 \mathrm{~Hz}, \mathrm{H}-10)$ to $\delta_{\mathrm{C}} 37.6(\mathrm{~d}, \mathrm{C}-6), 40.5$ $(\mathrm{d}, \mathrm{C}-9)$ and $66.9(\mathrm{t}, \mathrm{C}-11)$, indicated a hydroxy group at $\mathrm{C}-11$. In addition, the connections of C-8/C-3, C-3/C-4, C-3/C-2, and C-3/C-13 were deduced from HMBC correlations from $\delta_{\mathrm{H}} 1.05(3 \mathrm{H}, \mathrm{s}, \mathrm{H}-8)$ to $\delta_{\mathrm{C}} 43.0(\mathrm{~s}, \mathrm{C}-3), 39.9(\mathrm{t}, \mathrm{C}-4), 156.8(\mathrm{~s}, \mathrm{C}-2)$, and $58.2(\mathrm{~d}, \mathrm{C}-13)$. Moreover, the proton of an oxygenated methine at $\delta_{\mathrm{H}} 4.02(\mathrm{H}, \mathrm{d}, J=5.9 \mathrm{~Hz}, \mathrm{H}-14)$ showed key correlations to C-13, C-3, and $\delta_{\mathrm{C}} 42.2(\mathrm{~d}, \mathrm{C}-7)$, which indicated that $\delta_{\mathrm{C}} 69.6(\mathrm{~d}, \mathrm{C}-14)$ should be placed at $\mathrm{C}-13$. The above $2 \mathrm{D}$ NMR data analysis suggested that compound $\mathbf{1}$ possessed a sativene type sesquiterpene backbone. A ROESY experiment was carried out to establish the relative configuration of 1 (Figure 3). The key correlations of $\mathrm{H}-13 / \mathrm{H}-8, \mathrm{H}-13 / \mathrm{H}-6$, $\mathrm{H}-8 / \mathrm{H}-14$, and $\mathrm{H}-7 / \mathrm{H}-13$ suggested that $\mathrm{H}-6, \mathrm{H}-7, \mathrm{H}-8$, and $\mathrm{H}-13$ were $\beta$ oriented, while the correlation of $\mathrm{H}-1 / \mathrm{H}-9$ indicated that $\mathrm{H}-1$ and $\mathrm{H}-9$ were $\alpha$-oriented. Because of the rigid structure and the ROESY correlation of $\mathrm{H}-8 / \mathrm{H}-14$, both $\mathrm{H}-14$ and $\mathrm{H}-15$ were assigned as an $\alpha$ orientation [31]. Finally, the single-crystal X-ray diffraction not only confirmed the planar structure, as elucidated above, but also established the absolute configuration of $\mathbf{1}$ (Flack parameter $=-0.10(7)$, CCDC: 2124305 ; Figure 4$)$.

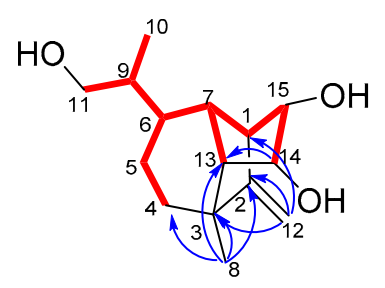

1

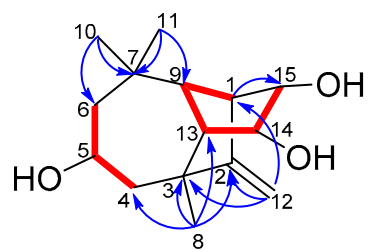

9

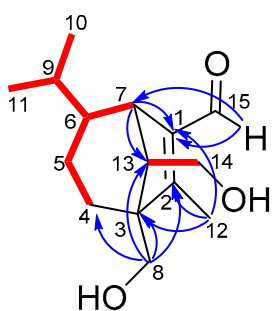

3

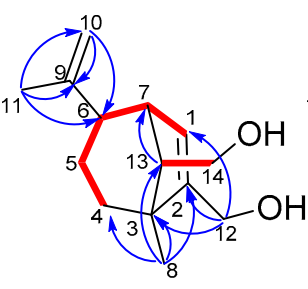

5

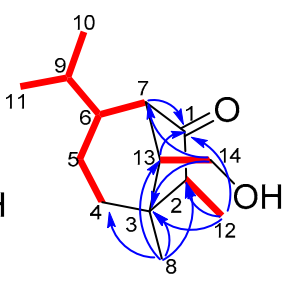

6

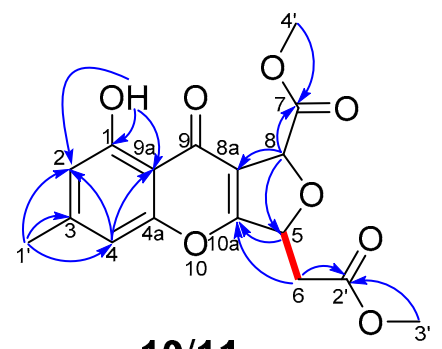

$10 / 11$

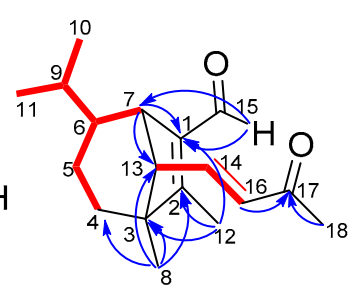

7

$\left[{ }^{1} \mathrm{H}-{ }^{1} \mathrm{H}\right.$ COSY

HMBC

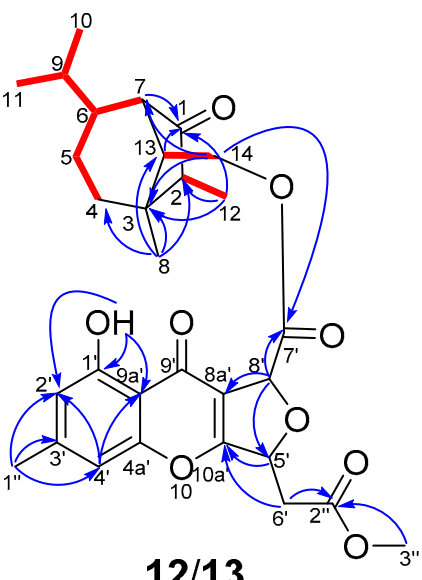

$12 / 13$

Figure 2. Key ${ }^{1} \mathrm{H}-{ }^{1} \mathrm{H}$ COSY and HMBC correlations for 1, 3, 5, 6, 7, and 9-13. 


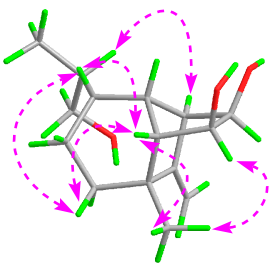

1

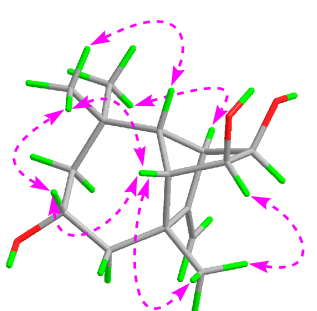

9

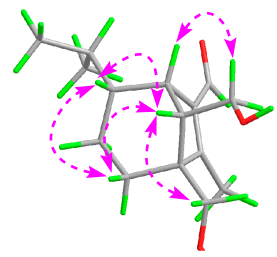

3

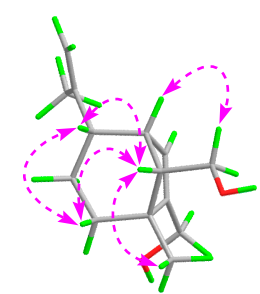

5

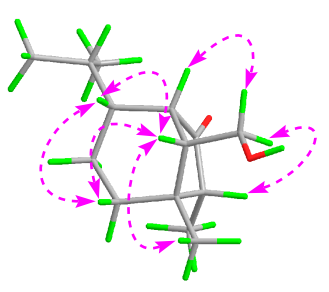

6

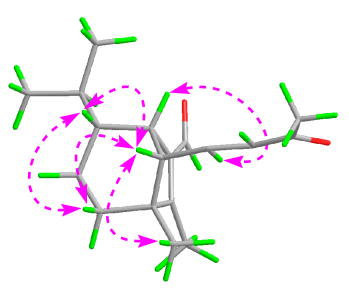

7

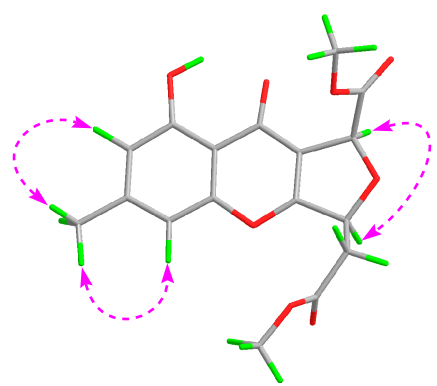

10

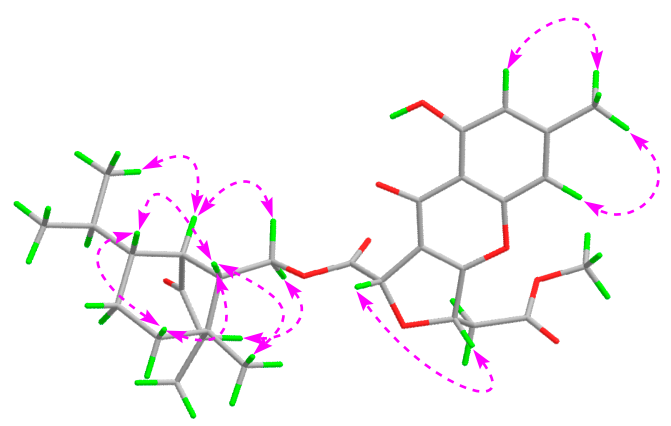

12

Figure 3. Key ROESY correlations for 1, 3, 5, 6, 7, 9, 10, and 12.

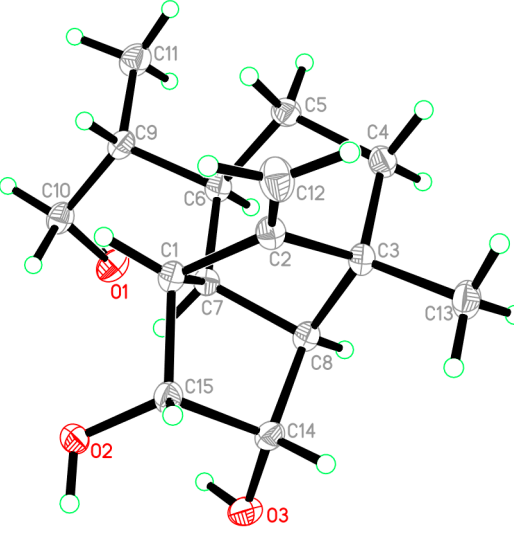

1

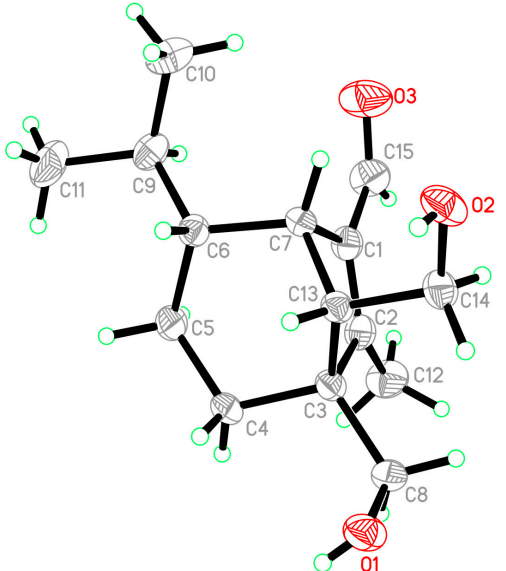

3

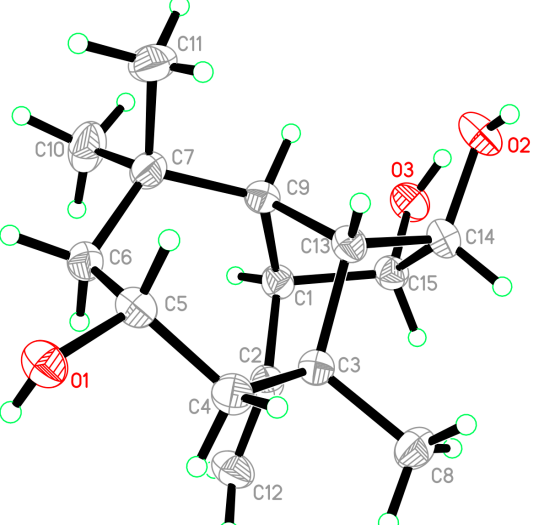

9

Figure 4. ORTEP diagrams of 1, 3, and 9.

The molecular formula of bipolarisorokin B (2) was determined to be $\mathrm{C}_{15} \mathrm{H}_{24} \mathrm{O}_{3}$ from the HRESIMS data (measured at $m / z 275.16166[\mathrm{M}+\mathrm{Na}]^{+}$, calculated for $\mathrm{C}_{15} \mathrm{H}_{24} \mathrm{O}_{3} \mathrm{Na}^{+}$ 275.16177). Close similarities were observed in the 1D NMR data (Table 1) of compound 1. However, signals for a methyl $\left(\delta_{\mathrm{H}} 0.94, \mathrm{~d}, J=6.9 \mathrm{~Hz}, \mathrm{H}-11 ; \delta_{\mathrm{C}} 16.4, \mathrm{C}-11\right)$ and an oxygenated quaternary carbon $\left(\delta_{\mathrm{C}} 73.7, \mathrm{C}-6\right)$ in 2 was suggested to replace the oxymethylene $\left(\delta_{\mathrm{H}} 3.64\right.$, overlap, $\left.\mathrm{H}-11 ; \delta_{\mathrm{C}} 66.9, \mathrm{C}-11\right)$ and the methine $\left(\delta_{\mathrm{H}} 1.65, \mathrm{~m}, \mathrm{H}-6 ; \delta_{\mathrm{C}} 37.6, \mathrm{C}-6\right)$ in 1 . These observations indicated that the hydroxy group at C-10 in $\mathbf{1}$ migrated to C-6 in 2 . The observed ${ }^{1} \mathrm{H}-{ }^{1} \mathrm{H}$ COSY cross-peak of $\mathrm{H}-10\left(\delta_{\mathrm{H}} 0.88,3 \mathrm{H}, \mathrm{d}, J=6.9 \mathrm{~Hz}\right)$ and $\mathrm{H}-9\left(\delta_{\mathrm{H}} 1.57\right.$, $1 \mathrm{H}, \mathrm{m})$, and $\mathrm{H}-9 / \mathrm{H}-11$, along with the HMBC correlations from $\mathrm{H}-10$ to C-6, C-9, and C-11 confirmed the above deduction (Figure 2). Furthermore, ROESY correlations of $\mathrm{H}-13 / \mathrm{H}-8$, $\mathrm{H}-8 / \mathrm{H}-14, \mathrm{H}-7 / \mathrm{H}-13$, and $\mathrm{H}-1 / \mathrm{H}-9$ revealed that compounds 2 and $\mathbf{1}$ shared the same relative configuration. In consideration of its biosynthetic origin, the absolute configuration of compound $\mathbf{2}$ was identified the same as that of $\mathbf{1}$. 
Bipolarisorokin C (3) was obtained as colorless needles. Its molecular formula of $\mathrm{C}_{15} \mathrm{H}_{24} \mathrm{O}_{3}$ was determined on the basis of the HR-ESIMS data (measured at $\mathrm{m} / \mathrm{z} 253.17971$ $[\mathrm{M}+\mathrm{H}]^{+}$, calculated for $\left.\mathrm{C}_{15} \mathrm{H}_{25} \mathrm{O}_{3}{ }^{+} 253.17982\right)$, corresponding to four degrees of unsaturation. The ${ }^{1} \mathrm{H}$ NMR data (Table 1) showed characteristic signals, including three methyls at $\delta_{\mathrm{H}} 0.78(3 \mathrm{H}, \mathrm{d}, J=6.4 \mathrm{~Hz}, \mathrm{H}-10), 1.06(3 \mathrm{H}, \mathrm{d}, J=6.4 \mathrm{~Hz}, \mathrm{H}-11)$, and $2.13(3 \mathrm{H}, \mathrm{s}, \mathrm{H}-12)$, and the proton of an aldehyde group at $\delta_{\mathrm{H}} 10.02(\mathrm{H}, \mathrm{s}, \mathrm{H}-15)$. The ${ }^{1} \mathrm{H}$ and ${ }^{13} \mathrm{C}$ NMR data, in association with the HSQC data, revealed three methyls, four methenes, five methines, and three nonprotonated carbons (Table 1). Preliminary analyses on the 1D NMR data revealed that 3 was likely to be a seco-sativene type sesquiterpenoid. Detailed analyses of the 2D NMR data indicated that the majority of the data of $\mathbf{3}$ was the same as those of helminthosporol [32], except for a hydroxy group at C-8 $\left(t, \delta_{\mathrm{C}} 64.6\right)$ in 3 , which was confirmed by the HMBC correlations from $\delta_{\mathrm{H}} 3.63(\mathrm{H}, \mathrm{d}, J=11.6 \mathrm{~Hz}, \mathrm{H}-8 \mathrm{a})$ and $3.71(\mathrm{H}$, $\mathrm{d}, J=11.6 \mathrm{~Hz}, \mathrm{H}-8 \mathrm{~b})$ to $\delta_{\mathrm{C}} 58.0$ (s, C-3), 29.6 (t, C-4), 167.0 (s, C-2), and 60.8 (d, C-13) (Figure 2). A ROESY experiment was carried out to establish the relative configuration of 3 (Figure 3). The cross peaks of H-13/H-8a, H-13/H-4b, H-4b/H-6, and H-7/H-14b were observed, which indicated that $\mathrm{H}-6, \mathrm{H}-7, \mathrm{H}-8$, and $\mathrm{H}-13$ were $\beta$ oriented. Furthermore, single crystal $X$-ray diffraction established the relative configuration (Flack parameter $=$ $-0.40(17)$, CCDC: 2124306; Figure 4), and the absolute configuration of 3 was determined by ECD calculations, as shown in Figure 5.

Bipolarisorokin D (4) was isolated as a colorless oil. The molecular formula was determined to be $\mathrm{C}_{15} \mathrm{H}_{24} \mathrm{O}_{3}$ according to the HRESIMS spectra (measured at $\mathrm{m} / \mathrm{z} 275.16153$ $[\mathrm{M}+\mathrm{Na}]^{+}$, calculated for $\left.\mathrm{C}_{15} \mathrm{H}_{24} \mathrm{NaO}_{3}{ }^{+} 275.16177\right)$. Compound 4 had the same molecular formula and NMR spectral patterns to that of 3 (Table 2). The key difference was an oxygenated quaternary carbon $\left(\delta_{\mathrm{C}} 73.5, \mathrm{~s}\right)$ in 4 instead of the methine in $3\left(\delta_{\mathrm{C}} 46.4, \mathrm{~d}\right)$. The HMBC correlations from H-4a $\left(\delta_{\mathrm{H}} 1.38, \mathrm{~m}\right), \mathrm{H}-5 \mathrm{~b}\left(\delta_{\mathrm{H}} 1.61, \mathrm{~m}\right), \mathrm{H}-7\left(\delta_{\mathrm{H}} 3.16, \mathrm{br} \mathrm{s}\right), \mathrm{H}-10\left(\delta_{\mathrm{H}}\right.$ $1.02, \mathrm{~d}, J=6.6 \mathrm{~Hz})$, and $\mathrm{H}-11\left(\delta_{\mathrm{H}} 0.80, \mathrm{~d}, J=6.6 \mathrm{~Hz}\right)$ to $\delta_{\mathrm{C}} 73.5$ established the quaternary carbon to be C-6. In addition, a methyl $\left(\mathrm{s}, \delta_{\mathrm{H}} 1.07, \mathrm{H}-8 ; \delta_{\mathrm{C}} 18.7, \mathrm{C}-8\right)$ in 4 replaced the oxygenated methylene $\left(\delta_{\mathrm{C}} 64.6\right)$ of $\mathrm{C}-8$ in 3 , which was verified by HMBC correlations from $\mathrm{H}-8\left(\delta_{\mathrm{H}} 1.07, \mathrm{~s}\right)$ to $\mathrm{C}-2\left(\delta_{\mathrm{C}} 170.4, \mathrm{~s}\right), \mathrm{C}-3\left(\delta_{\mathrm{C}} 52.0, \mathrm{~s}\right), \mathrm{C}-4\left(\delta_{\mathrm{C}} 32.4, \mathrm{t}\right)$, and $\mathrm{C}-13\left(\delta_{\mathrm{C}} 55.3, \mathrm{~d}\right)$. Detailed analyses of 2D NMR (HSQC, HMBC, ${ }^{1} \mathrm{H}-{ }^{1} \mathrm{H}$ COSY and ROESY) data confirmed that the other fragments of 4 were the same as those of 3.

Bipolarisorokin E (5) was obtained as a colorless oil. Its molecular formula $\mathrm{C}_{14} \mathrm{H}_{22} \mathrm{O}_{2}$ was characterized according to HRESIMS (measured at $m / z 221.15529[\mathrm{M}-\mathrm{H}]^{-}$, calculated for $\left.\mathrm{C}_{14} \mathrm{H}_{21} \mathrm{O}_{2}{ }^{-} 221.15470\right)$, implying four degrees of unsaturation. The general features of its NMR data closely resembled that of 3 (Table 2). Detailed analyses of 1D and 2D NMR data revealed the differences. At first, the loss of the aldehyde group at C-1 was revealed by the chemical shift of $\mathrm{C}-1$ at $\delta_{\mathrm{C}} 124.2$, along with the data from ${ }^{1} \mathrm{H}-{ }^{1} \mathrm{H}$ COSY and HMBC spectra as shown in Figure 2. Second, the hydroxy migrated from C-8 to C-12 $\left(\delta_{C} 59.8, t\right)$ as identified by the HMBC correlation from $\delta_{\mathrm{H}} 4.06(2 \mathrm{H}, \mathrm{m}, \mathrm{H}-12)$ to $\delta_{\mathrm{C}} 124.2(\mathrm{~d}, \mathrm{C}-1), 147.2$ (s, C-2), and 47.7 (s, C-3). Third, one double bond between C-9 and C-10 was built by HMBC correlations from $\delta_{\mathrm{H}} 4.69(2 \mathrm{H}, \mathrm{d}, J=5.1 \mathrm{~Hz}, \mathrm{H}-10)$ to $\delta_{\mathrm{C}} 22.7(\mathrm{q}, \mathrm{C}-11)$ and $45.2(\mathrm{~d}, \mathrm{C}-6)$. The other parts of 5 were elucidated as the same as those of 3 by a detailed analysis of 2D NMR data.

Bipolarisorokin $\mathrm{F}(6)$ was purified as white powder, and its molecular formula $\mathrm{C}_{14} \mathrm{H}_{24} \mathrm{O}_{2}$ was determinded according to HRESIMS (measured at $m / z 225.18506[\mathrm{M}+\mathrm{H}]^{+}$, calculated for $\mathrm{C}_{14} \mathrm{H}_{25} \mathrm{O}_{2}{ }^{+} 225.18491$ ). Analyses of the $1 \mathrm{D}$ and 2D NMR data (Table 2) suggested that 6 showed structural similarities to 3 . The distinction between the two compounds was that the $\alpha, \beta$-unsaturated aldehyde group $\left(\delta_{\mathrm{C}} 140.0, \mathrm{C}-1 ; \delta_{\mathrm{C}} 167.0, \mathrm{C}-2 ; \delta_{\mathrm{C}} 190.0, \mathrm{C}-15\right)$ in 3 was replaced by a carbonyl $\left(\delta_{\mathrm{C}} 212.0, \mathrm{C}-1\right)$ and a methylene group $\left(\delta_{\mathrm{C}} 50.7, \mathrm{C}-2\right)$ in 6 . It was supported by HMBC correlations from $\delta_{\mathrm{H}} 2.70(\mathrm{H}$, br s, H-7), $0.96(3 \mathrm{H}, \mathrm{q}, J=7.2 \mathrm{~Hz}$, $\mathrm{H}-12)$, and $1.72(\mathrm{H}, \mathrm{dd}, J=7.9,5.0 \mathrm{~Hz}, \mathrm{H}-13)$ to $\delta_{\mathrm{C}} 212.0(\mathrm{~s}, \mathrm{C}-1), 50.7(\mathrm{~d}, \mathrm{C}-2)$, and the COSY cross-peak of $\delta_{\mathrm{H}} 2.10(1 \mathrm{H}, \mathrm{m}, \mathrm{H}-2)$ and $\mathrm{H}-12$. The hydroxymethyl group $(\mathrm{C}-8)$ in 3 was replaced by a methyl group at C-8 $\left(\delta_{\mathrm{C}} 22.1, \mathrm{q}\right)$ in 6 , as well as the HMBC correlations from $\delta_{\mathrm{H}} 1.09(3 \mathrm{H}, \mathrm{s}, \mathrm{H}-8)$ to $\mathrm{C}-2, \delta_{\mathrm{C}} 41.8(\mathrm{~s}, \mathrm{C}-3), \delta_{\mathrm{C}} 36.1(\mathrm{t}, \mathrm{C}-4)$, and $\delta_{\mathrm{C}} 54.9(\mathrm{~d}, \mathrm{C}-13)$. The key 
ROESY cross-peak (Figure 3) of H-2/Ha- $14\left(\mathrm{H}, \mathrm{dd}, J=10.7,5.0 \mathrm{~Hz}, \delta_{\mathrm{H}} 3.85\right)$ suggested that $\mathrm{H}-2$ was $\beta$ oriented. Other ROESY data revealed the same patterns to 3 . Finally, regarding the same origin of $\mathbf{6}$ and $\mathbf{3}$, the absolute configuration of $\mathbf{6}$ was identified to be the same as that of 3 , as depicted.
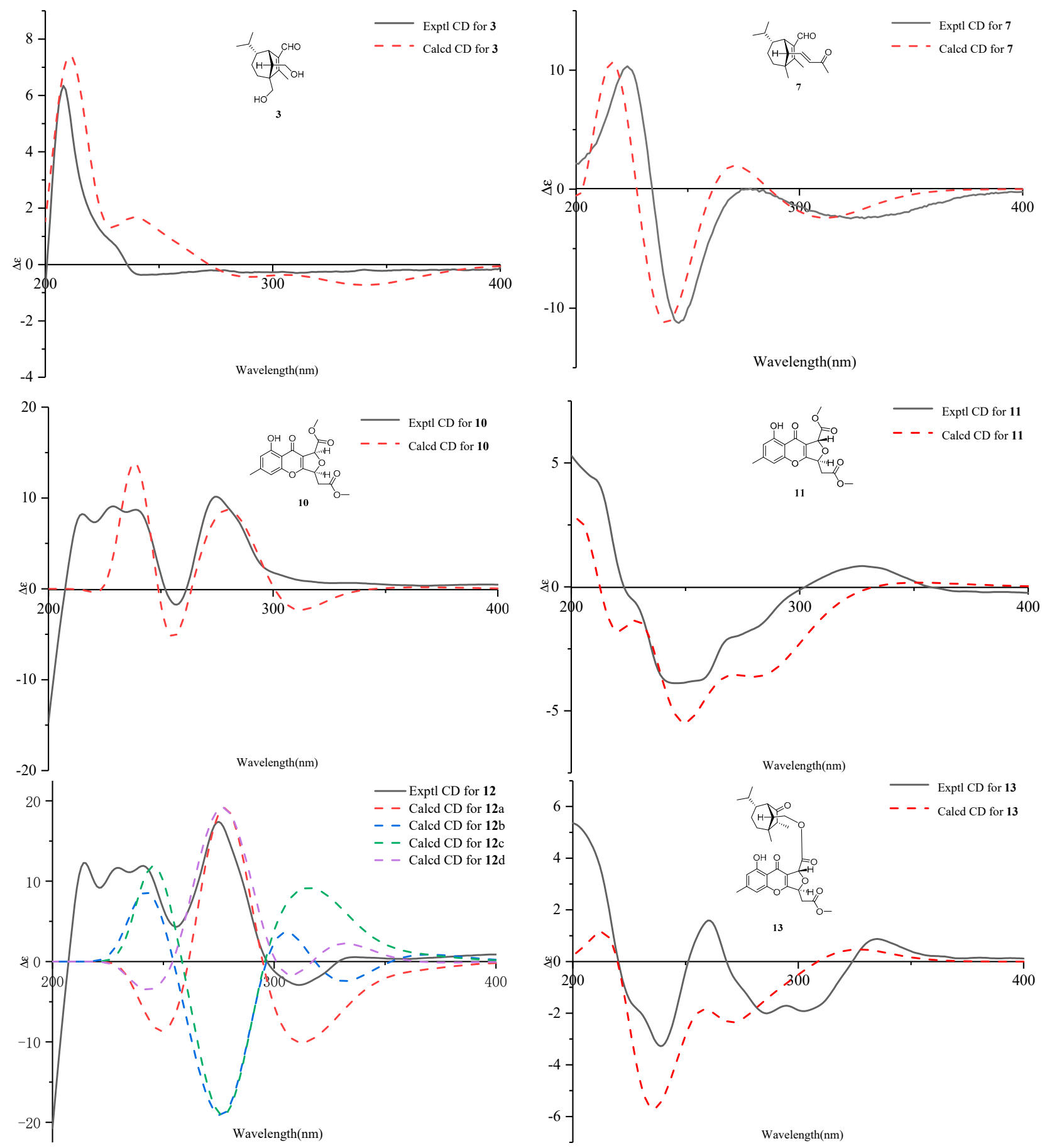

Figure 5. ECD calculations for 3, 7, and 10-13.

The molecular formula of bipolarisorokin $\mathrm{G}$ (7) was assigned as $\mathrm{C}_{18} \mathrm{H}_{26} \mathrm{O}_{2}$ based on its HRESIMS spectrum (measured at $m / z 275.20059[\mathrm{M}+\mathrm{H}]^{+}$, calculated for $\mathrm{C}_{18} \mathrm{H}_{27} \mathrm{O}_{2}{ }^{+}$, 275.20056), which contained three more carbon atoms than 3 . The interpretation of the ${ }^{1} \mathrm{H}$ and ${ }^{13} \mathrm{C}$ NMR data of 7 (Table 3) indicated the same structure skeleton to that of 3 . Analyses of 2D NMR spectra revealed modifications in 7 (Figure 2). HMBC correlations from $\delta_{\mathrm{H}}$ 
$0.97(3 \mathrm{H}, \mathrm{s}, \mathrm{H}-8)$ to $\delta_{\mathrm{C}} 165.3(\mathrm{~s}, \mathrm{C}-2), 52.6(\mathrm{~s}, \mathrm{C}-3), 33.7(\mathrm{t}, \mathrm{C}-4)$, and $63.6(\mathrm{~d}, \mathrm{C}-13)$ suggested that a hydroxy group was missing in 7 . In addition, an $\alpha, \beta$-unsaturated ketone group was identified by the HMBC correlations from $\delta_{\mathrm{H}} 6.55(\mathrm{H}, \mathrm{dd}, J=15.9,9.6 \mathrm{~Hz}, \mathrm{H}-14), 6.08(\mathrm{H}$, $\mathrm{d}, J=15.9 \mathrm{~Hz}, \mathrm{H}-16)$, and $2.20(3 \mathrm{H}, \mathrm{s}, \mathrm{H}-18)$ to $\delta_{\mathrm{C}} 198.6(\mathrm{~s}, \mathrm{C}-17)$. In the ${ }^{1} \mathrm{H}-{ }^{1} \mathrm{H}$ COSY spectrum, correlations from $\mathrm{H}-14$ to $\delta_{\mathrm{H}} 2.22(\mathrm{H}, \mathrm{d}, J=9.6 \mathrm{~Hz}, \mathrm{H}-13)$ and $\mathrm{H}-16$ indicated that the $\alpha, \beta$-unsaturated carboxyl moiety was located at C-13. Finally, the absolute configuration of 7 can be fully resolved by the ECD calculation, as shown in Figure 5.

Bipolarisorokin $\mathrm{H}(8)$ was obtained as a colorless oil. Its molecular formula, $\mathrm{C}_{17} \mathrm{H}_{24} \mathrm{O}_{3}$, was inferred from the pseudomolecular ion peak at $\mathrm{m} / \mathrm{z} 277.17984[\mathrm{M}+\mathrm{H}]^{+}$in the HRESIMS (calculated for $\mathrm{C}_{17} \mathrm{H}_{25} \mathrm{O}_{3}{ }^{+}$277.17982). The NMR data of 8 (Table 3) resembled that of 7, except for the presence of a carboxyl $\left(\delta_{C} 171.1, C-17\right)$ in 8 instead of a carbonyl $\left(\delta_{C} 198.6\right.$, $\mathrm{C}-17)$ in 7 , as well as the loss of a methyl group. This was supported by HMBC correlations from $\delta_{\mathrm{H}} 6.80(\mathrm{H}, \mathrm{dd}, J=15.4,9.9 \mathrm{~Hz}, \mathrm{H}-14)$ and $5.81(\mathrm{H}, \mathrm{d}, J=15.5 \mathrm{~Hz}, \mathrm{H}-16)$ to $\delta_{\mathrm{C}} 171.1(\mathrm{~s}$, $\mathrm{C}-17)$. Detailed analyses of 2D NMR data suggested that the other data were the same as those of 7 .

Bipolarisorokin I (9) was isolated as colorless crystals. Its molecular formula was identified as $\mathrm{C}_{15} \mathrm{H}_{24} \mathrm{O}_{3}$ by HRESIMS (measured at $\mathrm{m} / \mathrm{z} 251.16621[\mathrm{M}-\mathrm{H}]^{-}$, calculated for $\left.\mathrm{C}_{21} \mathrm{H}_{23} \mathrm{O}_{3}-251.16527\right)$. All the spectroscopic data indicated similar patterns to those of longifolene [33]. Detailed analyses of 1D and 2D NMR data revealed the differences. Signals at $\delta_{C} 67.0(\mathrm{~d}, \mathrm{C}-5), 70.5(\mathrm{~d}, \mathrm{C}-14)$, and $74.9(\mathrm{~d}, \mathrm{C}-15)$ were identified as the oxygenated methines. Therefore, three hydroxyls were suggested to be placed at C-5, C-14, and C-15, respectively, which were identified by the HMBC and ${ }^{1} \mathrm{H}-{ }^{1} \mathrm{H}$ COSY correlations, as shown in Figure 2. Comprehensive analyses of other data suggested that the other parts of 9 were the same as those of longifolene. The relative configuration of $\mathbf{9}$ was revealed by a ROESY experiment, as shown in Figure 3. The ROESY correlations of Me-10/H-13, H-13/H-5, Me-8/H-13, Me-10/H-9, and Me-10/H-5 indicated these groups were cofacial (assigned as $\beta$ orientation). In addition, the Me- $11 / \mathrm{H}-1$ interaction suggested that $\mathrm{H}-1$ should be $\alpha$ oriented. Moreover, the coupling constant between H-14 and H-15 $\left(J_{14,15}=6.2 \mathrm{~Hz}\right)$, as well as the ROESY correlations of Me-8/H-14 and Me-8/H-15, suggested that H-14 and H-15 were $\alpha$ oriented. Finally, the single-crystal X-ray diffraction not only confirmed the planar structure but also established the absolute configuration of 9 (Flack parameter $=0.01(3)$, CCDC: 2124307; Figure 4).

Bipolarithone A (10) was isolated as a yellow oil, and its molecular formula was determined to be $\mathrm{C}_{17} \mathrm{H}_{16} \mathrm{O}_{8}$ by HRESIMS (measured at $m / z 349.09143[\mathrm{M}+\mathrm{H}]^{+}$, calculated for $\mathrm{C}_{17} \mathrm{H}_{17} \mathrm{O}_{8}{ }^{+}$349.09179). The NMR data (Table 4) of $\mathbf{1 0}$ were similar to those of the dechlorinated methyl ester (16) isolated in this study [34]. The major difference was that 10 exhibited a dihydrofuran ring rather than a furan ring. HMBC correlations from $\mathrm{H}-8(\mathrm{H}, \mathrm{d}$, $\left.J=3.9 \mathrm{~Hz}, \delta_{\mathrm{H}} 5.64\right)$ to $\mathrm{C}-8 \mathrm{a}\left(\delta_{\mathrm{C}} 114.7, \mathrm{~s}\right), \mathrm{C}-7\left(\delta_{\mathrm{C}} 170.0, \mathrm{~s}\right), \mathrm{C}-9\left(\delta_{\mathrm{C}} 178.3, \mathrm{~s}\right)$, and C-10a $\left(\delta_{\mathrm{C}}\right.$ $167.7, \mathrm{~s})$, together with $\mathrm{H}-5\left(\mathrm{H}, \mathrm{ddd}, J=6.6,4.4,3.9 \mathrm{~Hz}, \delta_{\mathrm{H}} 5.73\right)$ to $\mathrm{C}-10 \mathrm{a}, \mathrm{C}-8 \mathrm{a}, \mathrm{C}-6\left(\delta_{\mathrm{C}} 37.7\right.$, t), and $C-2^{\prime}\left(\delta_{C} 169.5, s\right)$, supported the above assignment. The relative configuration of 10 was identified by the analysis of its ROESY data. The ROESY correlation between H-8 and $\mathrm{H}-5$ indicated that $\mathrm{H}-8$ had the same orientation as $\mathrm{H}-5$ (assigned as an $\alpha$ orientation). The calculated ECD of $\mathbf{1 0}$ established the configuration of 10, as shown in Figure 5. Therefore, the structure of $\mathbf{1 0}$ was characterized as depicted.

Bipolarithone B (11) was isolated as a yellow oil. The HRESIMS spectrum of 11 suggested a molecular formula of $\mathrm{C}_{17} \mathrm{H}_{16} \mathrm{O}_{8}$ (measured at $m / z 349.09157[\mathrm{M}+\mathrm{H}]^{+}$, calculated for $\mathrm{C}_{17} \mathrm{H}_{17} \mathrm{O}_{8}{ }^{+}$349.09179), the same as that of 10. The planar structure of $\mathbf{1 1}$ was elucidated to be the same as that of $\mathbf{1 0}$ by the analysis of its $1 \mathrm{D}$ and $2 \mathrm{D}$ NMR data. The main difference was suggested as its stereochemistry at C-8 $\left(\delta_{C} 79.8, d\right)$. Analyses of the ${ }^{1} \mathrm{H}$ NMR information showed that the coupling constants of $\mathrm{H}-8, \mathrm{H}-5$, and $\mathrm{H}-6$ were significantly different from those of 11, as shown in the Table 4. Furthermore, the ROESY correlation of $\mathrm{H}-8\left(\delta_{\mathrm{H}} 5.63,1 \mathrm{H}, \mathrm{d}, J=1.7 \mathrm{~Hz}\right) / \mathrm{H}-5\left(\delta_{\mathrm{H}} 5.62,1 \mathrm{H}, \mathrm{ddd}, J=8.4,3.8,1.7 \mathrm{~Hz}\right)$ was not observed in 11. These data suggested that $\mathbf{1 1}$ was an epimer of 10. The ECD calculation for 11 was 
performed, and the results of $\mathbf{1 1}$ matched well with the experimental ECD curve (Figure 5). Hence, the absolute configuration of $\mathbf{1 1}$ can be fully assigned, as shown.

Bipolarithone $\mathrm{C}$ (12) was assigned a molecular formula of $\mathrm{C}_{30} \mathrm{H}_{36} \mathrm{O}_{9}$ based on its HRESIMS data (measured at $m / z 541.24310[\mathrm{M}+\mathrm{H}]^{+}$, calculated for $\mathrm{C}_{30} \mathrm{H}_{37} \mathrm{O}_{9}{ }^{+} 541.24321$ ). The NMR data of $\mathbf{1 2}$ were very similar to those of bipolenin I (14) (Table 5), a novel sesquiterpenoid-xanthone adduct isolated from the fungus Bipolaris eleusines [35]. The significant differences were that there was an absence of an aldehyde group and two olefinic carbons, as well as the presence of an additional methine and carbonyl, in $\mathbf{1 2}$. These data suggested that the $\alpha, \beta$-unsaturated aldehyde moiety disappeared in $\mathbf{1 2}$. This assignment was confirmed by the HMBC correlations of $\delta_{\mathrm{H}} 2.16(\mathrm{H}, \mathrm{m}, \mathrm{H}-2), 1.29(\mathrm{H}, \mathrm{m}$, H-6), $2.56\left(1 \mathrm{H}\right.$, br s, H-7), $0.95(3 \mathrm{H}, \mathrm{d}, J=7.2 \mathrm{~Hz}, \mathrm{C}-12)$, and $1.90(\mathrm{H}, \mathrm{m}, \mathrm{H}-13)$ to $\delta_{\mathrm{C}} 50.6$ (d, C-2) and 221.6 (s, C-1). The ROESY spectrum displayed similar patterns to those of 14. Furthermore, a cross peak between $\mathrm{H}-2$ and $\mathrm{H}-14 \mathrm{a}\left(\delta_{\mathrm{H}} 4.05,1 \mathrm{H}, \mathrm{dd}, J=11.3,5.1 \mathrm{~Hz}\right)$ confirmed the relative configuration of $\mathrm{C}-2$, as shown. The absolute configuration of $\mathbf{1 2}$ was elucidated by the quantum chemistry calculations. At first, the ECD calculations were conducted on the four possible conformers $(\mathbf{1 2 a}-\mathrm{d})$, using time-dependent density functional theory (TDDFT) at the B3LYP / 6-311G (d) level in methanol with the PCM model. The overall calculated ECD spectrum of each configuration was then generated according to the Boltzmann weighting of the conformers. As a result, the calculated ECD curves of 12a and 12d matched well with the experimental data (Figure 5). To determine its final structure, the theoretical NMR calculations and DP4+ probabilities were employed. The ${ }^{13} \mathrm{C}$ NMR chemical shifts of $\mathbf{1 2 a}$ and $\mathbf{1 2 d}$ were calculated at the mPW1PW91/6-311+G $(\mathrm{d}, \mathrm{p})$ level in the gas phase. According to the DP4+ probability analyses, 12a was assigned with $100 \%$ probability (see data in the Supporting Information). Structurally, compound $\mathbf{1 2}$ comprised of a seco-sativene sesquiterpenoid unit and a xanthone unit, whose absolute configurations were in accord with compound 6 and compound 10, respectively. Therefore, the structure of $\mathbf{1 2}$ was established as depicted.

Bipolarithone D (13) had the same molecular formula $\left(\mathrm{C}_{30} \mathrm{H}_{36} \mathrm{O}_{9}\right)$ as that of $\mathbf{1 2}$, according to their HRESIMS spectra (measured at $m / z 541.24316[\mathrm{M}+\mathrm{H}]^{+}$, calculated for $\left.\mathrm{C}_{30} \mathrm{H}_{37} \mathrm{O}_{9}+541.24321\right)$. The NMR resonances for 13 (Table 5) resembled those of 12, except that the resonances of $\mathrm{C}-6^{\prime}\left(\Delta \delta_{\mathrm{C}}+1.5\right), \mathrm{H}-6^{\prime} \mathrm{a}\left(\Delta \delta_{\mathrm{H}}+0.08\right)$, and $\mathrm{H}-6^{\prime} \mathrm{b}\left(\Delta \delta_{\mathrm{H}}+0.15\right)$ were shifted downfield, while the data $\mathrm{H}-5^{\prime}\left(\Delta \delta_{\mathrm{H}}-0.08\right)$ were shifted upfield. A detailed comparison of the 1D and 2D NMR data of $\mathbf{1 3}$ with that of $\mathbf{1 2}$ indicated that the two compounds possessed the same planar structure. The main difference was the stereochemistry at C- $8^{\prime}$. A key ROESY correlation of $\mathrm{H}-5^{\prime} / \mathrm{H}-8^{\prime}$ could be detected in $\mathbf{1 2}$ but not in 13. In addition, the coupling constants of $\mathrm{H}-8^{\prime}$ in $\mathbf{1 3}(\mathrm{J}=1.8 \mathrm{~Hz})$ were different from that in $\mathbf{1 2}(\mathrm{J}=3.9 \mathrm{~Hz})$. All the data suggested that compound $\mathbf{1 3}$ was a C-8 ${ }^{\prime}$ epimer of $\mathbf{1 2}$. Finally, the absolute configuration of 13 was confirmed by ECD calculations (Figure 5).

Five known compounds were determined as bipolenins I and J (14 and 15), dechlorinated methyl ester (16), drechslerines A (17), and (+)-secolongifolene diol (18) by the comparison of their spectral data with that reported in the literature $[32,34,35]$. In this study, the absolute configurations of compounds $\mathbf{1 7}$ and $\mathbf{1 8}$ were confirmed by single crystal X-ray diffractions (Figure 6), which could support the absolute configurations of 1-9, 12, and 13 as depicted in the text, since they were obtained from the same source.

All compounds (1-18) were evaluated for their anti-Psa activity. As a result, compounds 10 and 15 showed significant inhibitory activity, with MICs of 64 and $16 \mu \mathrm{g} / \mathrm{mL}$, respectively, while compounds 7, 11, 13, and 16 showed moderate activity, with MICs of $128 \mu \mathrm{g} / \mathrm{mL}$ (Table 6). 


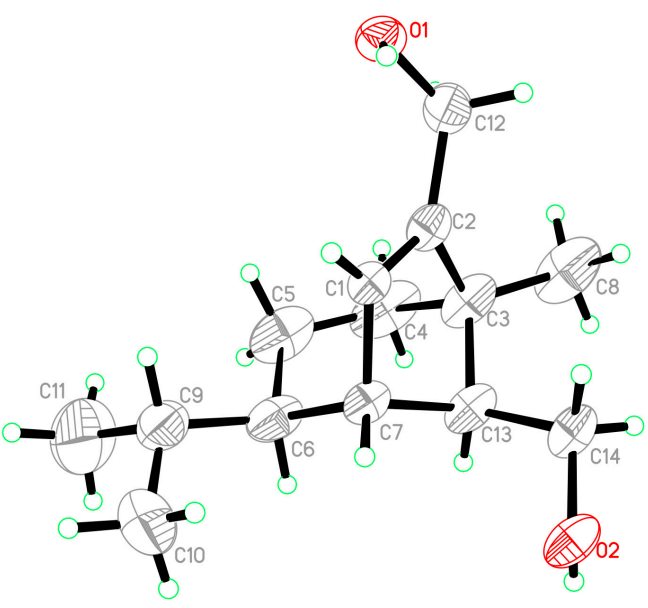

17

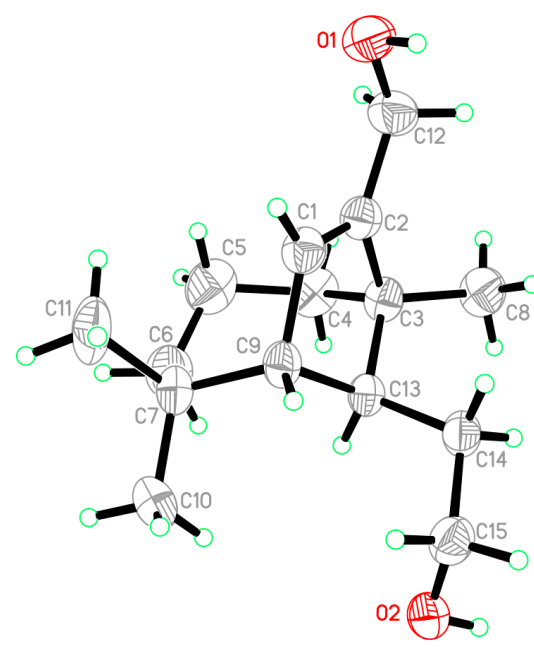

18

Figure 6. ORTEP diagrams of $\mathbf{1 7}$ and $\mathbf{1 8 .}$

Table 6. Inhibitory effects of the isolates against five plant pathogens (MIC, $\mu \mathrm{g} / \mathrm{mL}){ }^{\mathrm{a}}$.

\begin{tabular}{|c|c|c|c|c|c|}
\hline Compd & Psa & P. infestans & A. solani & R. solani & F. oxysporum \\
\hline 3 & 256 & NA & 128 & 256 & NA \\
\hline 4 & $\mathrm{NA}^{\mathrm{c}}$ & 128 & NA & NA & NA \\
\hline 7 & 128 & NA & 64 & 128 & 256 \\
\hline 8 & 256 & NA & 256 & NA & NA \\
\hline 9 & NA & 128 & NA & NA & NA \\
\hline 10 & 64 & 128 & NA & NA & NA \\
\hline 11 & 128 & 64 & NA & NA & NA \\
\hline 12 & 256 & 64 & NA & 64 & NA \\
\hline 13 & 128 & 32 & NA & NA & NA \\
\hline 14 & NA & NA & 8 & NA & 128 \\
\hline 15 & 16 & NA & 16 & NA & NA \\
\hline 16 & 128 & 128 & 128 & 256 & NA \\
\hline Streptomycin b & 8 & - & - & - & - \\
\hline Hygromycin $\mathrm{B}^{\mathrm{b}}$ & - & 8 & 4 & 16 & 32 \\
\hline
\end{tabular}

${ }^{a}$ Compounds without any bioactivity are not listed; ${ }^{\mathrm{b}}$ Positive controls; ${ }^{\mathrm{c}} \mathrm{NA}=$ no activity at $256 \mu \mathrm{g} / \mathrm{mL}$.

In addition, our previous study on chemicals from B. eleusines suggested that sativenexanthone adducts have promising inhibitory activity against plant pathogenic microorganisms [35]. Therefore, all compounds were evaluated for their inhibitory activity against four plant pathogenic microorganisms, including P. infestane, A. solani, R. solani, and F. oxysporum. As a result, many compounds showed certain inhibitory activity, as given in Table 6 .

A brief structure-activity relationship analysis suggested that the aldehyde-containing sativene sesquiterpenoids were more active than the others, while the xanthones or their derivatives showed better inhibitory activities than sativene sesquiterpenoids.

\section{Conclusions}

A total of 18 compounds, including 13 new ones, were characterized from the kiwifruitassociated fungus Bipolaris sp. Their structures, with absolute configurations, were established by means of spectroscopic methods. Many compounds possessed anti-Psa activity and inhibitory activity against plant pathogens. It is concluded that Bipolaris sp. is rich in sativene sesquiterpenoids and xanthones, and both sativene sesquiterpenoids and xanthones possess potential antimicrobial application prospects. This study also suggested that it is an effective way to find natural anti-Psa agents from kiwifruit-associated fungi. 
Supplementary Materials: The following are available online at https:/ /www.mdpi.com/article/10 .3390/jof8010009/s1, Section S1: Supplementary of NMR, HRESIMS and CD spectra for 1-13, Section S2: Calculational details for 3, 7, 10, 11, 12 and 13

Author Contributions: Conceptualization, J.H.; methodology, J.-J.Y., Y.-X.J., S.-S.H. and J.H.; resources, J.H.; data curation, J.-J.Y.; writing-original draft preparation, J.-J.Y.; writing-review and editing, J.H.; project administration, J.H.; funding acquisition, J.H. All authors have read and agreed to the published version of the manuscript.

Funding: This research was funded by the National Natural Science Foundation of China (grant number 22177139).

Institutional Review Board Statement: Not applicable.

Informed Consent Statement: Not applicable.

Data Availability Statement: X-ray crystallographic data of 1, 3, 9, and 18 (CIF) is available free of charge at https:/ / www.ccdc.cam.ac.uk (accessed on 1 December 2021).

Acknowledgments: The authors thank the Analytical \& Measuring Centre, South-Central University for Nationalities for the spectra measurements.

Conflicts of Interest: The authors declare no conflict of interest.

\section{References}

1. Dolly, S.; Kaur, J.; Bhadariya, V.; Sharma, K. Actinidia deliciosa (Kiwi fruit): A comprehensive review on the nutritional composition, health benefits, traditional utilization and commercialization. J. Food Process. Preserv. 2021, 45, e15588. [CrossRef]

2. Wang, S.N.; Qiu, Y.; Zhu, F. Kiwifruit (Actinidia spp.): A review of chemical diversity and biological activities. Food Chem. 2021, 350, 128469. [CrossRef]

3. Richardson, D.P.; Ansell, J.; Drummond, L.N. The nutritional and health attributes of kiwifruit: A review. Eur. J. Nutr. 2018, 57, 2659-2676. [CrossRef] [PubMed]

4. Wang, Q.; Zhang, C.; Long, Y.; Wu, X.; Su, Y.; Lei, Y.; Ai, Q. Bioactivity and control efficacy of the novel antibiotic tetramycin against various kiwifruit diseases. Antibiotics 2021, 10, 289. [CrossRef] [PubMed]

5. Kim, G.H.; Jung, J.S.; Koh, Y.J. Occurrence and epidemics of bacterial canker of kiwifruit in Korea. Plant Pathol. J. 2017, 33, 351-361. [CrossRef] [PubMed]

6. Wang, Q.; Zhang, C.; Wu, X.; Long, Y.; Su, Y. Chitosan augments tetramycin against soft rot in kiwifruit and enhances its improvement for kiwifruit growth, quality and aroma. Biomolecules 2021, 11, 1257. [CrossRef]

7. Lee, Y.S.; Han, H.S.; Kim, G.H.; Koh, Y.J.; Hur, J.S.; Jung, J.S. Causal agents of blossom blight of kiwifruit in Korea. Plant Pathol. J. 2009, 25, 220-224. [CrossRef]

8. Balestra, G.M.; Mazzaglia, A.; Rossetti, A. Outbreak of bacterial blossom blight caused by Pseudomonas viridiflava on Actinidia chinensis kiwifruit plants in Italy. Plant Dis. 2008, 92, 1707. [CrossRef]

9. Jeong, I.H.; Lim, M.T.; Kim, G.H.; Han, T.W.; Kim, H.C.; Kim, M.J.; Park, H.S.; Shin, S.H.; Hur, J.S.; Shin, J.S.; et al. Incidences of leaf spots and blights on kiwifruit in Korea. Plant Pathol. J. 2008, 24, 125-130. [CrossRef]

10. Li, H.H.; Tang, W.; Liu, K.; Zhang, L.; Tang, X.F.; Miao, M.; Liu, Y.S. First report of Fusarium fujikuroi causing brown leaf spot on kiwifruit. Plant Dis. 2020, 104, 1560. [CrossRef]

11. Polat, Z.; Awan, Q.N.; Hussain, M.; Akgul, D.S. First report of Phytopythium vexans causing root and collar rot of kiwifruit in Turkey. Plant Dis. 2017, 101, 1058. [CrossRef]

12. Wang, K.X.; Xie, Y.L.; Yuan, G.Q.; Li, Q.Q.; Lin, W. First report of root and collar rot caused by Phytopythium helicoides on kiwifruit (Actinidia chinensis). Plant Dis. 2015, 99, 725. [CrossRef]

13. McCann, H.C.; Li, L.; Liu, Y.F.; Li, D.W.; Pan, H.; Zhong, C.H.; Rikkerink, E.H.A.; Templeton, M.D.; Straub, C.; Colombi, E.; et al. Origin and evolution of the kiwifruit canker pandemic. Genome Biol. Evol. 2017, 9, 932-944. [CrossRef]

14. Vanneste, J.L. The scientific, economic, and social impacts of the New Zealand outbreak of bacterial canker of kiwifruit (Pseudomonas syringae pv. actinidiae). Annu. Rev. Phytopathol. 2017, 55, 377-399. [CrossRef]

15. Wicaksono, W.A.; Jones, E.E.; Casonato, S.; Monk, J.; Ridgway, H.J. Biological control of Pseudomonas syringae pv. actinidiae (Psa), the causal agent of bacterial canker of kiwifruit, using endophytic bacteria recovered from a medicinal plant. Biol. Control 2018, 116, 103-112. [CrossRef]

16. Scortichini, M. Aspects still to solve for the management of kiwifruit bacterial canker caused by Pseudomonas syringae pv. actinidiae biovar 3. Eur. J. Hortic. Sci. 2018, 83, 205-211. [CrossRef]

17. Bardas, G.A.; Veloukas, T.; Koutita, O.; Karaoglanidis, G.S. Multiple resistance of botrytis cinerea from kiwifruit to SDHIs, QoIs and fungicides of other chemical groups. Pest Manag. Sci. 2010, 66, 967-973. [CrossRef] [PubMed] 
18. Colombi, E.; Straub, C.; Kunzel, S.; Templeton, M.D.; McCann, H.C.; Rainey, P.B. Evolution of copper resistance in the kiwifruit pathogen Pseudomonas syringae pv. actinidiae through acquisition of integrative conjugative elements and plasmids. Environ. Microbiol. 2017, 19, 819-832. [CrossRef]

19. Gupta, S.; Chaturvedi, P.; Kulkarni, M.G.; van Staden, J. A critical review on exploiting the pharmaceutical potential of plant endophytic fungi. Biotechnol. Adv. 2020, 39, 107462. [CrossRef]

20. Kusari, S.; Hertweck, C.; Spitellert, M. Chemical ecology of endophytic fungi: Origins of secondary metabolites. Chem. Biol. 2012, 19, 792-798. [CrossRef]

21. Strobel, G.; Daisy, B.; Castillo, U.; Harper, J. Natural products from endophytic microorganisms. J. Nat. Prod. 2004, 67, 257-268. [CrossRef] [PubMed]

22. Helaly, S.E.; Thongbai, B.; Stadler, M. Diversity of biologically active secondary metabolites from endophytic and saprotrophic fungi of the ascomycete order Xylariales. Nat. Prod. Rep. 2018, 35, 992-1014. [CrossRef]

23. Zhang, J.Y.; He, J.; Li, Z.H.; Feng, T.; Liu, J.K. Zopfiellasins A-D, two pairs of epimeric cytochalasins from kiwi-associated fungus Zopfiella sp. and their antibacterial assessment. Molecules 2021, 26, 5611. [CrossRef]

24. Yi, X.W.; He, J.; Sun, L.T.; Liu, J.K.; Wang, G.K.; Feng, T. 3-Decalinoyltetramic acids from kiwi-associated fungus Zopfiella sp. and their antibacterial activity against Pseudomonas syringae. RSC Adv. 2021, 11, 18827-18831. [CrossRef]

25. Ma, J.T.; Du, J.X.; Zhang, Y.; Liu, J.K.; Feng, T.; He, J. Natural imidazole alkaloids as antibacterial agents against Pseudomonas syringae pv. actinidiae isolated from kiwi endophytic fungus Fusarium tricinctum. Fitoterapia 2022, 156, 105070. [CrossRef]

26. Frisch, M.J.T.; Trucks, G.W.; Schlegel, H.B.; Scuseria, G.E.; Robb, M.A.; Cheeseman, J.R.; Scalmani, G.; Barone, V.; Mennucci, B.; Petersson, G.A.; et al. Gaussian 09; Revision D. 01; Gaussian Inc.: Wallingford, CT, USA, 2010.

27. Shao, Y.; Molnar, L.F.; Jung, Y.; Kussmann, J.; Ochsenfeld, C.; Brown, S.T.; Gilbert, A.T.B.; Slipchenko, L.V.; Levchenko, S.V.; O'Neill, D.P.; et al. Advances in methods and algorithms in a modern quantum chemistry program package. Phys. Chem. Chem. Phys. 2006, 8, 3172-3191. [CrossRef] [PubMed]

28. Hehre, W.J. A Guide to Molecular Mechanics and Quantum Chemical Calculations; Wavefunction Inc.: Irvine, CA, USA, 2003; Volume 51, pp. 1-812. [CrossRef]

29. Bruhn, T.; Schaumlöffel, A.; Hemberger, Y.; Bringmann, G. SpecDis: Quantifying the comparison of calculated and experimental electronic circular dichroism spectra. Chirality 2013, 25, 243-249. [CrossRef] [PubMed]

30. Grimblat, N.; Zanardi, M.M.; Sarotti, A.M. Beyond DP4: An improved probability for the stereochemical assignment of isomeric compounds using quantum chemical calculations of NMR shifts. J. Org. Chem. 2015, 80, 12526-12534. [CrossRef] [PubMed]

31. Nukina, M.; Hattori, H.; Marumo, S. Cis-Sativenediol, a plant growth promotor, produced by fungi. J. Am. Chem. Soc. 1975, 97, 2542-2543. [CrossRef]

32. Osterhage, C.; König, G.M.; Höller, U.; Wright, A.D. Rare sesquiterpenes from the algicolous fungus Drechslera dematioidea. J. Nat. Prod. 2002, 65, 306-313. [CrossRef]

33. Dorn, F.; Arigoni, D. Ein bicyclischer Abkömmling von (-) longifolen aus Helminthosporium sativum und H. victoriae. Experientia 1974, 30, 851-852. [CrossRef]

34. Han, J.; Zhang, J.; Song, Z.; Liu, M.; Hu, J.; Hou, C.; Zhu, G.; Jiang, L.; Xia, X.; Quinn, R.; et al. Genome- and MS-based mining of antibacterial chlorinated chromones and xanthones from the phytopathogenic fungus Bipolaris sorokiniana strain 11134. Appl. Microbiol. Biotechnol. 2019, 103, 5167-5181. [CrossRef] [PubMed]

35. He, J.; Yang, M.S.; Wang, W.X.; Li, Z.H.; Elkhateeb, W.; Wen, T.C.; Ai, H.L.; Feng, T. Anti-phytopathogenic sesquiterpenoidxanthone adducts from potato endophytic fungus Bipolaris eleusines. RSC Adv. 2019, 9, 128-131. [CrossRef] 